\title{
Relation of Electroencephalogram to Changes in Blood Flow in Response to Brain Stimulation
}

\author{
Satoshi Sanada \\ Department of Physiology, Kansai Medical University, \\ Moriguchi, Osaka, Japan \\ (Supervised by Prof. Motohiro Yasuhara, M.D.)
}

\section{Introduction}

Since the times of Roy and Scherrington ${ }^{1)}$, many reports have been written dealing with relations between the cerebral blood flow and electrical activity of the brain. In the present study, the mechanisms of changes in cephalic blood flow (carotid, vertebral and regional cortical blood flow) have been pursued in terms of electrophysiology in the presence of arousal reaction, recruiting response, spike and wave and hippocampal seizure. Special reference was made of the hippocampus as to EEG pattern during stimulation of the mesencephalic reticular formation (MRF) and cephalic blood flow in consideration of changes in intracranial pressure (ICP).

As to the pattern of hippocampal EEG, since the first striking report by Green and Arduini $^{2}$ ' in which it was revealed that synchronized, rhythmic activity in the hippocampus occurred in response to various sensory and arousing stimuli, a number of studies have been attempted to reveal the mechanism of theta rhythm activity and desynchronization $\left(\right.$ Stumpf $^{3)}$, Yokota and Fujimori4), Anchel and Lindsley ${ }^{5)}$, Paiva et $\mathrm{al}^{(6)}$, and Klemm ${ }^{7)}$ ). As to the relations between pattern of hippocampal EEG and autonomic activity, Torii ${ }^{8)}$ described that fast wave is concerned with the parasympathetic activity and that slow wave is concerned with sympathetic activity, based upon the results of arterial blood pressure. Yamamoto et $\mathrm{al}^{91}$ reported that the hippocampus (HPC) had something to do with sympathetic activity based upon the results of the experiment in which they observed the relationship between hippocampal EEG and the nictitating membrane in cats. In the present study, the relationship between hippocampal EEG and cephalic blood flow (carotid, vertebral and regional cortical blood flow) was examined.

In order to elucidate the effect of central nervous activity on peripheral circulation, experiments of cervical sympathetic nerve cut, stimulation and impairment of interpeduncular nucleus (IPN), administration of atropine and stimulation of MRF and HPC were also performed. 
For the measurement of the blood flow, electromagnetic blood flowmeter and crossed thermocouple tissue blood flowmeter were used in the present study. Actually, there are many other methods to measure cerebral and cephalic blood flow. Skull window technique and drop count method are classical ones and the following are commonly used today. They are electromagnetic blood flowmeter (to measure the internal carotid blood flow, the common carotid blood flow after the ligation of the external carotid artery, the cerebral venous outflow and the vertebral blood flow), crossed thermocouple tissue blood flowmeter, Doppler blood flowmeter, photo-electric method, nitrous oxide clearance method, hydrogen clearance method, ${ }^{85} \mathrm{Kr}$ clearance method, ${ }^{133}$ Xe clearance method and heat clearance method. Every method has its merits and demerits. Therefore we must carefully select the most suitable one. Consequently electromagnetic blood flowmeter and crossed thermocouple tissue blood flowmeter were used to obtain them.

\section{Methods}

Rabbits weighing from 2 to $2.5 \mathrm{~kg}$ were used without anesthesia. After tracheostomy and canulation, each animal was placed in a stereotaxic apparatus (Todai-Noken), with the scalp incised and a large part of the skull removed. The common carotid arteries were exposed unilaterally (in 3 cases, the external carotid arteries were ligated at their origins). In the former half from results $A$ to $G$, the common carotid arteries were bluntly dissected for $45 \mathrm{~mm}$ in length, in order to fix the probe firmly according to the individual method of $U_{c h i y a m a}{ }^{10)}$. In the latter half from results $H$ to $T$, the common carotid arteries were bluntly dissected for $8 \mathrm{~mm}$ in length, paying attention not to injure the cervical sympathetic nerve and the probe was fixed by use of wires, paying keen attention to the angle of probe to the common carotid artery. The vertebral arteries were exposed unilaterally in 11 cases. The femoral arteries were exposed bilaterally. One is for measurement of blood flow and another is for arterial pressure. For measurement of carotid, vertebral and femoral blood flow, Nihonkoden electromagnetic flowmeter (MF-26) was used and blood flow volume was calculated by use of an integrator. A probe of an electromagnetic flowmeter, $1.5 \mathrm{~mm}$ in diameter, was used for measurement of carotid blood flow. A probe of an electromagnetic flowmeter, $1.0 \mathrm{~mm}$ in diameter, was used for measurement of vertebral and femoral blood flow. For measurement of cortical blood flow, Unique Medical crossed thermocouple tissue blood flowmeter was used. A probe of a crossed thermocouple tissue blood flowmeter was placed extradurally on the parietal cortex.

For electrical stimulation, Nihonkoden stimulator (MSE-3R) was used. In all cases, 
the stimulation pulses were $1 \mathrm{msec}$ in duration and were presented at rate of 100,8 and $3 \mathrm{~Hz}$. As stimulating electrodes in the brain, bipolar stainless-steel electrodes $(250 \mu$ in diameter), each bared $0.5 \mathrm{~mm}$ at the tips and with bared regions separated by $0.5 \mathrm{~mm}$, were used. According to the atlas of Sawyer ${ }^{11)}$, electrodes were inserted in the mesencephalic reticular formation (MRF), the hippocampus (HPC), the amygdala (AMYG) and the nucleus ventralis anterior of thalamus (VA). These electrodes were fixed to the skull with dental cement. Recordings from the frontal cortex were made with disc electrodes (10mm in diameter). Recordings from the hippocampus were made with bipolar electrodes. EMG was induced from flexor muscle (M. articuloulnaris) of the fore limb and extensor muscle (M. quadriceps femoris) of the hind limb. All recordings were made with Sanei (EG-900) electroencephalograph and Nihonkoden Medicalcorder. Interpedunclar nucleus (IPN) electrolytic lesions were made by passing a DC current (40 volt for $30 \mathrm{sec}$ ). EEG, EMG, ECG, arterial pressure and blood flow were recorded simultaneously at a speed of $15 \mathrm{~mm}$ per second.

Intracranial pressure was measured and altered by connecting an intraventricularly inserted tube to a pressure sensitive transducer. For analysis of blood gases, blood was sampled from a femoral artery. Arterial pressure was measured continuously from a femoral artery. Pentobarbital, metrazol and atropine were administrated from an auricular vein. At the end of the experiment the brain was removed and fixed in 10 per cent formalin. The locations of the electrodes were determined by the examination of serial sections.

\section{Results}

A. Effect of MRF stimulation on carotid and femoral blood flow

It is well-known that MRF stimulation at high frequency produces an arousal reaction in the cerebral cortex and evoked muscular discharge in the fore and hind limbs, during which period carotid and femoral blood flow were measured.

Fig. 1 shows the changes in EEG, carotid and femoral blood flow, arterial blood pressure, EMG and ECG due to MRF stimulation at $100 \mathrm{~Hz}, 1.4 \mathrm{~V}$. Arousal reaction in the cerebral cortex and evoked muscular discharge in the fore and hind limbs were produced by MRF stimulation (heavy line). One to two seconds after the beginning of stimulation, carotid blood flow began to increase. Near the end of stimulation, it reached the peak, then returned to prestimulation level 10 seconds after the end of stimulation. When blood flow increases, wave of integration becomes dense. Femoral blood flow began to increase 3 to 4 seconds after the beginning of stimulation. Time 


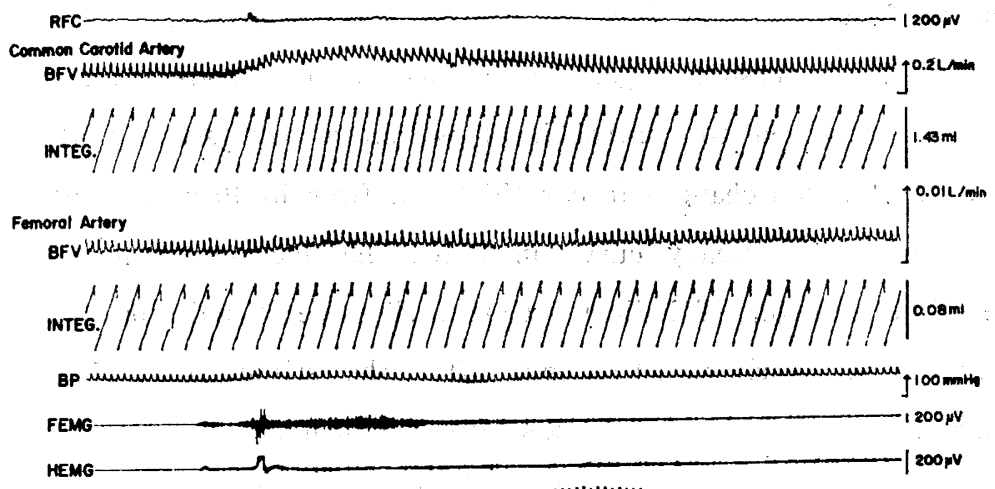

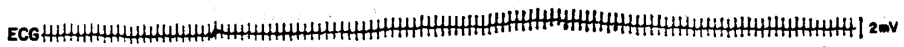

AF $100 \mathrm{~Hz} 1.4 \mathrm{~V}$

$\sqrt{1000}$

Fig. 1. Effect of MRF stimulation on EEG, carotid blood flow, femoral blood flow, arterial blood pressure, EMG and ECG. Heavy line indicates period of stimulation. Abbreviations : $\mathrm{RFC}=\mathrm{EEG}$ induced from right frontal cortex; $\mathrm{BFV}=$ blood flow volume ; INTEG. =integration ; $\mathrm{BP}=$ arterial blood pressure; $\mathrm{FEMG}=\mathrm{EMG}$ induced from fore limb; HEMG=EMG induced from hind limb.

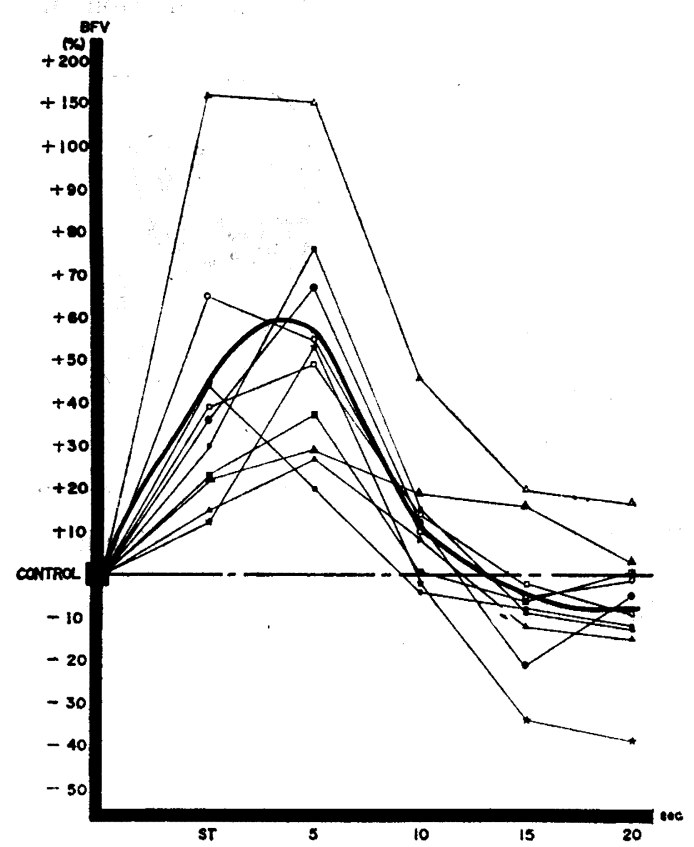

Fig. 2. Effect of MRF stimulation on carotid blood flow. Heavy curve indicates the average in 10 cases.

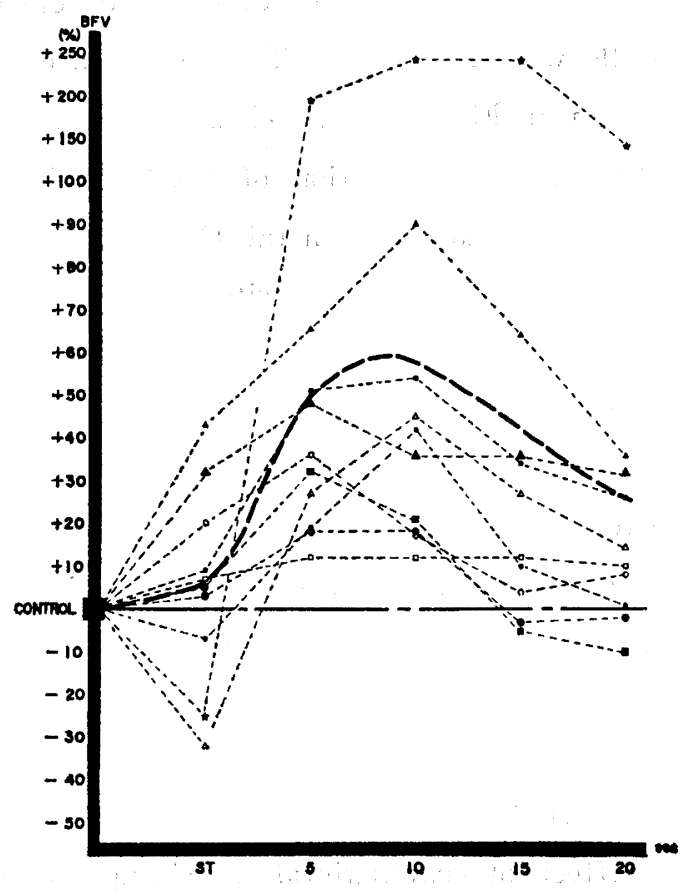

Fig. 3. Effect of MRF stimulation on femoral blood flow. 
of regression in femoral blood flow to control level was longer than that in carotid blood flow. Arterial pressure began to increase two seconds after the beginning of stimulation, then returned to control level within a few seconds after the end of stimulation. Fig. 2 shows changes in carotid blood flow in 10 cases that were collected from these experiments. Heavy curve indicates the average in 10 cases. Horizontal axis indicates time passage, ST indicates time during the stimulation, 5 shows 5 seconds after the stimulation, 10 shows 10 seconds after the stimulation, 15 shows 15 seconds after the stimulation, 20 shows 20 seconds after the stimulation. Vertical axis indicates ratio of variation in blood flow compared with prestimulation level. The peak time of increased blood flow was between ST and 5. Blood flow increased by $60 \%$ at maximum in average, and it returned to control level from 10 to 15 seconds after the end of stimulation. Fig. 3 shows changes in femoral blood flow in 10 cases. MRF stimulation at $100 \mathrm{~Hz}$ caused a reduction in femoral blood flow in 3 out of 10 cases. In 7 out of 10 cases, blood flow increased. After the stimulation, in both cases, blood flow increased rapidly, and the increased ratio reached the peak 5 to 10 seconds after the end of stimulation. Time of regression in femoral blood flow to control level was longer than that in carotid blood flow. Thus, there were two patterns of the femoral blood flow in response to MRF stimulation. One is considered to decrease and the other to increase. Therefore, in order to elucidate the cause, histological examination in MRF was performed. The results are shown in Fig. 4. Solid circle indicates the site of stimulation of MRF which caused increase in femoral blood flow. Open circle indicates the site of stimulation of MRF which caused the femoral blood flow to reduce. Based upon these results, it could be understood that the sites where the reduction was caused were concentrated on the center of the mesencephalic reticular formation in plate P. 8 according to Sawyer's atlas.
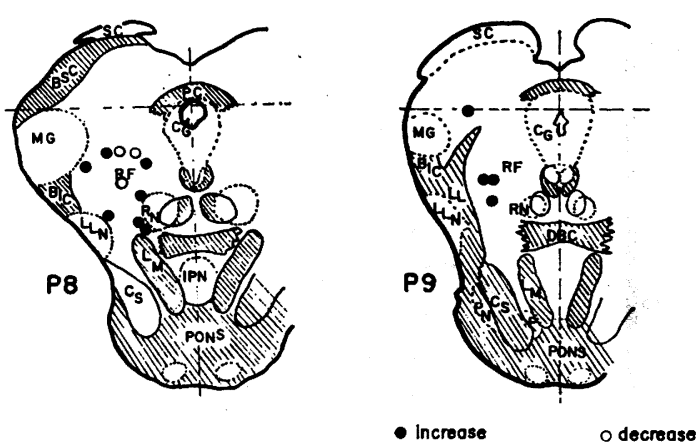

Fig. 4. Histological aspect of MRF stimulation. Abbreviations : RF= reticular formation; $I P N=N$. interpeduncularis ; $L M=$ Medial lemniscus; $\mathrm{RN}=$ Red nucleus ; $\mathrm{CG}=$ Central grey.

B. Effect of pentobarbital on changes in carotid and femoral blood flow due to MRF stimulation

Effect of pentobarbital on changes in carotid and femoral blood flow due to MRF stimulation at $100 \mathrm{~Hz}$ was examined. The results were shown in Fig. 5. Straight line 

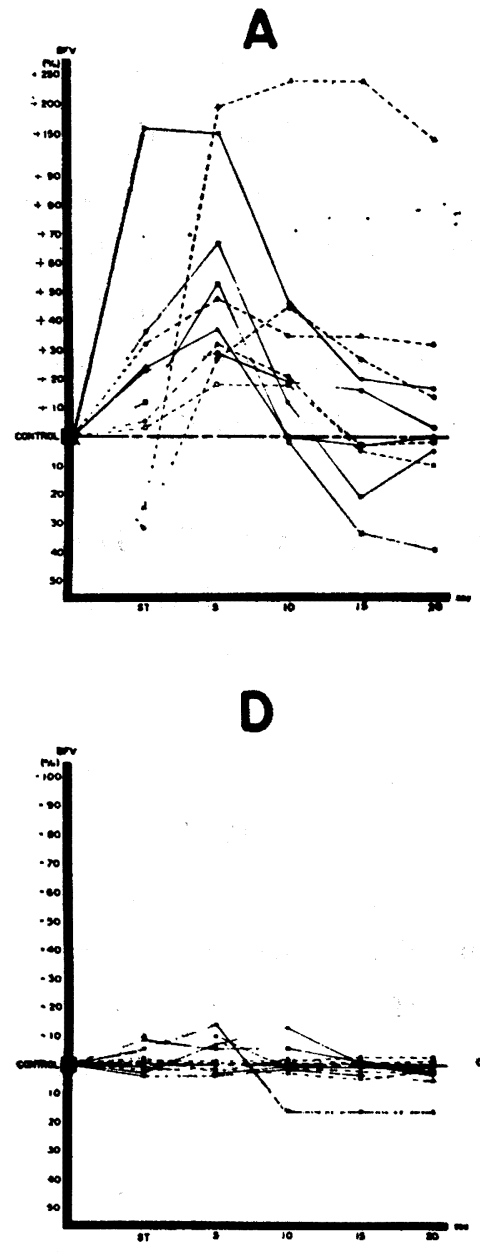

B

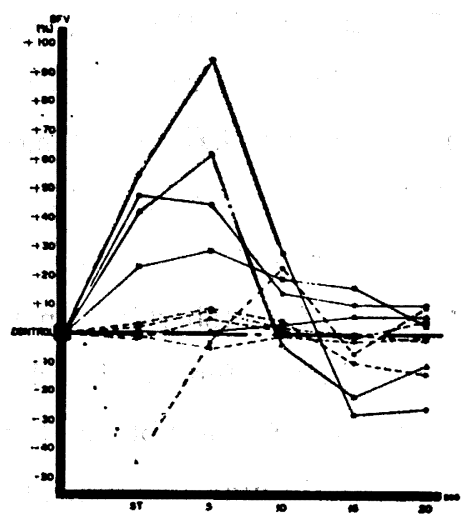

$E$

B

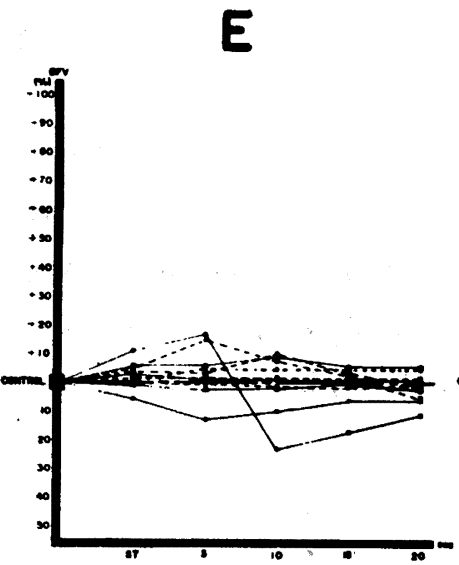

C

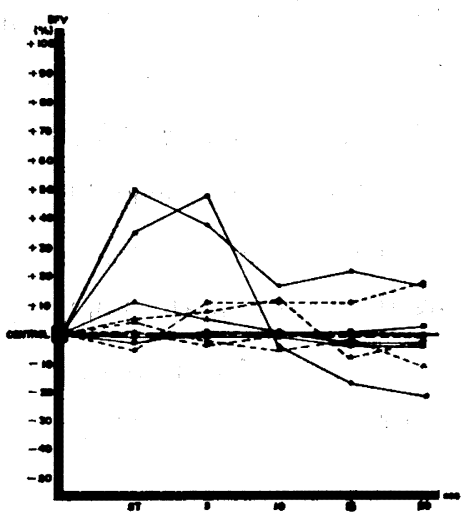

F

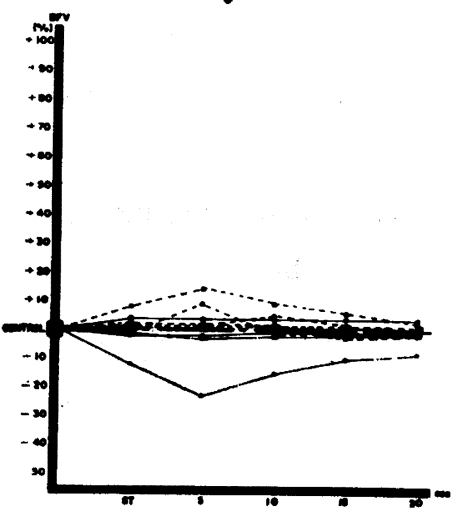

Fig. 5. Effect of pentobarbital on changes in carotid blood flow $(-)$ and femoral blood flow (......) due to MRF stimulation at $100 \mathrm{~Hz}$.

indicates changes in carotid blood flow. Dashed line indicates changes in femoral blood flow. Fig. 5, A indicates the changes in carotid and femoral blood flow due to MRF stimulation before the administration of pentobarbital. Fig. 5, B indicates the changes in blood flow 3 minutes after the administration of $1 \mathrm{mg} / \mathrm{kg}$ pentobarbital. Fig. 5, C is identical with Fig. 5, B after the administration of $3 \mathrm{mg} / \mathrm{kg}$ pentobarbital. Fig. 5, D is identical with Fig. 5, B after the administration of $5 \mathrm{mg} / \mathrm{kg}$ pentobarbital. Fig. $\mathbf{5}, \mathbf{E}$ is identical with Fig. 5, B after the administration of $10 \mathrm{mg} / \mathrm{kg}$ pentobarbital. Fig. 5, F is identical with Fig. 5, B after the administration of $20 \mathrm{mg} / \mathrm{kg}$ pentobarbital. Following the administration of $1 \mathrm{mg} / \mathrm{kg}$ pentobarbital in Fig. 5, B, femoral blood flow stopped changing except for one case, and the increased ratio in carotid blood flow became 
lower. Following the administration of $3 \mathrm{mg} / \mathrm{kg}$ pentobarbital in Fig. 5, C, femoral blood flow stopped changing in all cases, and the increased ratio in carotid blood flow turned to as low as $10 \%$ in 3 out of 5 cases, and in the rest, carotid blood flow continued to increase showing approximately $50 \%$ in its increased ratio. Following the administration of $5 \mathrm{mg} / \mathrm{kg}$ in Fig. 5, D, $10 \mathrm{mg} / \mathrm{kg}$ in Fig. $5, \mathbf{E}$ and $20 \mathrm{mg} / \mathrm{kg}$ in Fig. 5, F pentobarbital, both femoral and carotid blood flow stopped changing in spite of MRF stimulation. Based upon these results, the inhibition on carotid ane femoral blood flow could be considered as the result of the inhibitory effect of pentobarbital on the reticular formation.

C. Effect of pentobarbital on carotid and femoral blood flow

Effect of pentobarbital on carotid and femoral blood flow was examined. Fig. 6 shows the changes in carotid blood flow. Horizontal axis indicates dosage of pentobarbital, and vertical axis indicates ratio of variation in blood flow compared with prestimulation level. Following the administration of $1 \mathrm{mg} / \mathrm{kg}$ to $10 \mathrm{mg} / \mathrm{kg}$ pentobarbital, numbers of increased and decreased cases were even. Following the administration of $20 \mathrm{mg} / \mathrm{kg}$ pentobarbital, the cases of increased blood flow became predominant. Fig. 7 shows the changes in femoral blood flow. Following the administration of such a small amount as $1 \mathrm{mg} / \mathrm{kg}$ and $3 \mathrm{mg} / \mathrm{kg}$ pentobarbital, in 3 out of 8 cases, blood flow decreased, and in the rest, blood flow increased. Following the administration of such a great amount as $10 \mathrm{mg} / \mathrm{kg}$ and $20 \mathrm{mg} / \mathrm{kg}$ pentobarbital, blood flow increased by $40 \%$ and $75 \%$ respectively

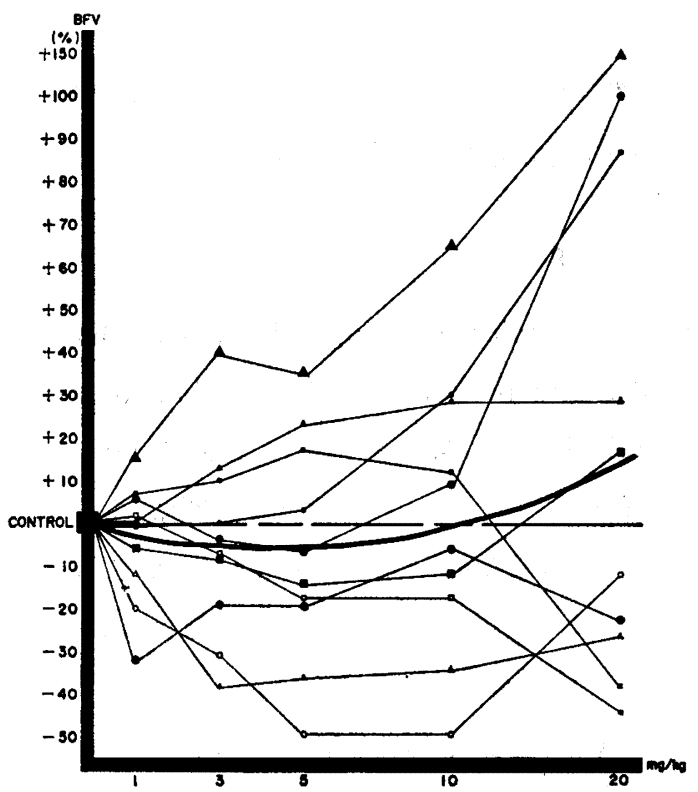

Fig. 6. Effect of pentobarbital on carotid blood flow.

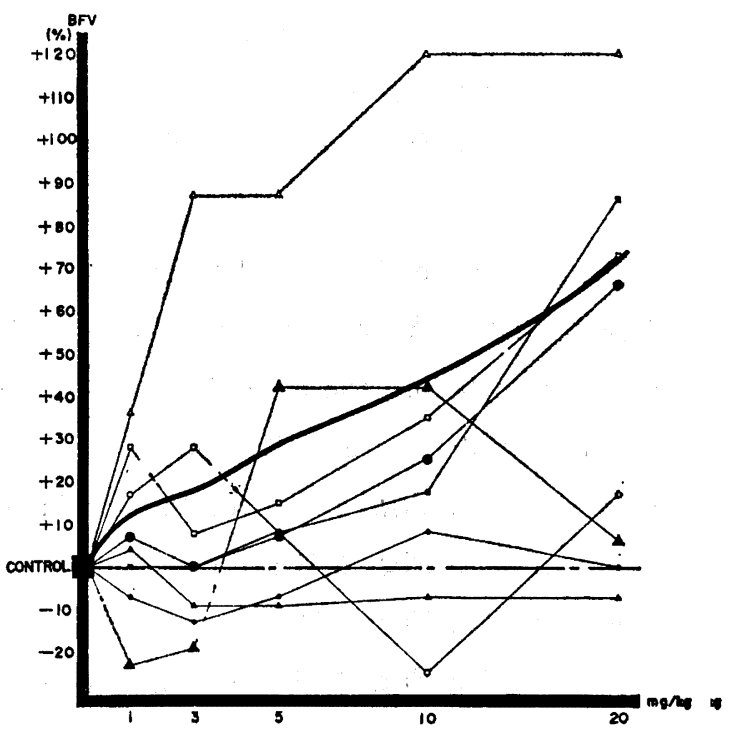

Fig. 7. Effect of pentobarbital on femoral blood flow. 
in average. Arterial pressure decreased by $20 \%$ to $30 \%$ and $R R$ interval prolonged following the administration of $20 \mathrm{mg} / \mathrm{kg}$ pentobarbital. Thus as above described, the effect of barbiturate, which inhibits the activity of the reticular formation, was examined. Then, effect of metrazol, which is the central nervous system stimulant, was examined.

D. Effect of metrazol on changes in carotid and femoral blood flow due to MRF stimulation

Fig. 8, $\mathbf{A}$ shows the changes in carotid blood flow (straight line) and femoral blood flow (dashed line) due to MRF stimulation at $100 \mathrm{~Hz}$. Fig. 8, B indicates the changes in blood flow due to MRF stimulation 3 minutes after the administration of $1 \mathrm{mg} / \mathrm{kg}$ metrazol. Fig, 8, C is identical with Fig. 8, B after the administration of $3 \mathrm{mg} / \mathrm{kg}$ metrazol. Fig. $\mathbf{8}, \mathrm{D}$ is identical with Fig. 8, B after the administration of

A

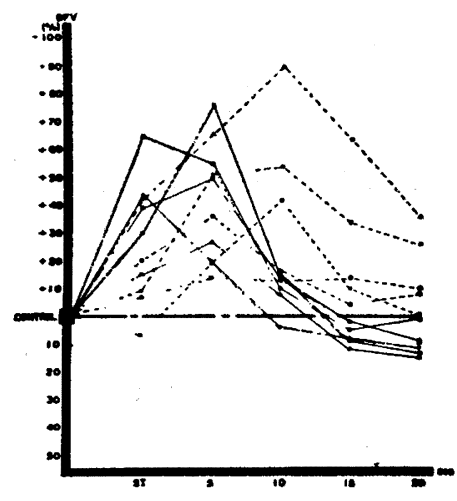

D

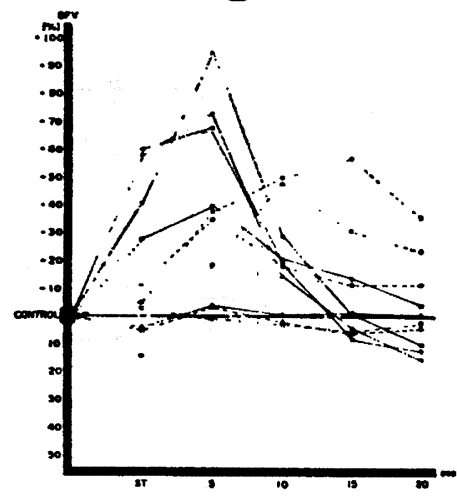

B

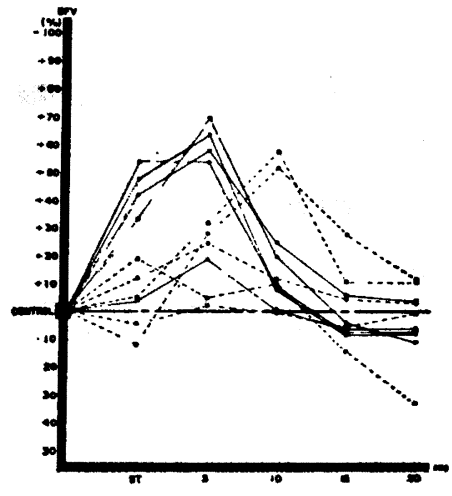

$\mathbf{E}$

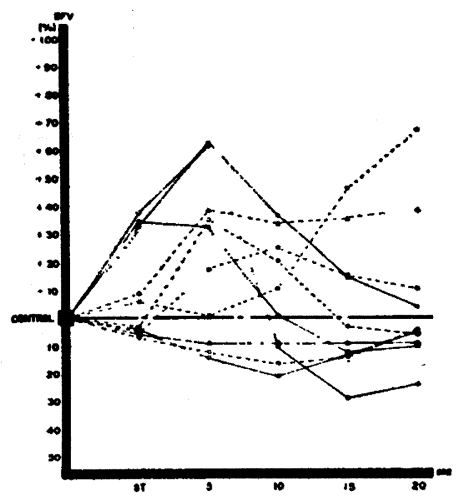

C

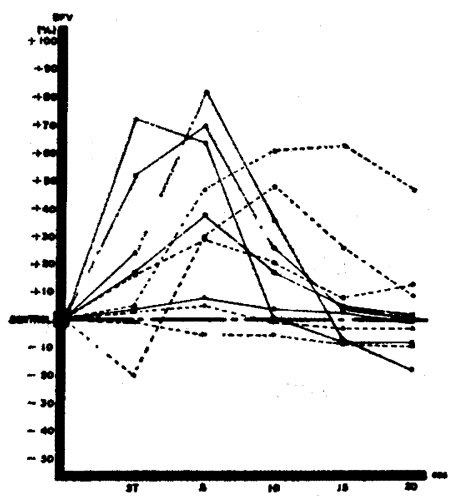

F

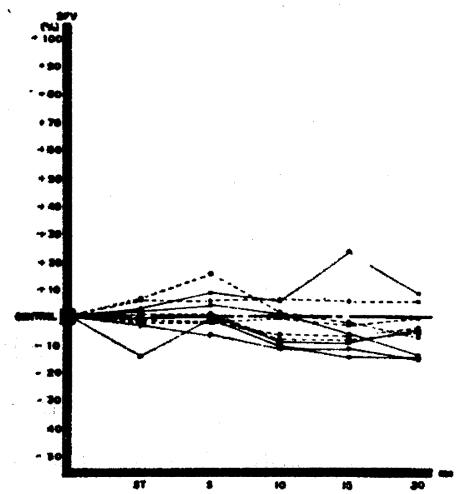

Fig. 8. Effect of metrazol on changes in carotid blood flow $(-)$ and femoral blood flow (…..) due to MRF stimulation at $100 \mathrm{~Hz}$. 
$5 \mathrm{mg} / \mathrm{kg}$ metrazol. Fig. 8, $\mathrm{E}$ is identical with Fig. 8, B after the administration of $10 \mathrm{mg} / \mathrm{kg}$ metrazol. Fig. 8, F is identical with Fig. 8, B after the administration of $20 \mathrm{mg} / \mathrm{kg}$ metrazol. Following the administration of $3 \mathrm{mg} / \mathrm{kg}$ in Fig. 8, C and $5 \mathrm{mg} / \mathrm{kg}$ in Fig. 8, D metrazol, the increased ratio in carotid blood flow increased. There was a case, for instance, in which carotid blood flow showed such a remarkable increase as $100 \%$ after the administration of $5 \mathrm{mg} / \mathrm{kg}$ metrazol in Fig. 8, D. On the contrary, there was a case, in which carotid blood flow decreased. Following the administration of $10 \mathrm{mg} / \mathrm{kg}$ metrazol in Fig. 8, E, the increased ratio slightly decreased, and there were two cases with decreased blood flow. Following the administration of $20 \mathrm{mg} / \mathrm{kg}$ metrazol in Fig. 8, F, carotid blood flow stopped changing in spite of MRF stimulation. On the other hand, the increased ratio in femoral blood flow became lower with the increase of the dosage of metrazol. Following the administration of $20 \mathrm{mg} / \mathrm{kg} \mathrm{me-}$ trazol, femoral blood flow stopped changing, which is similar to the changes in carotid blood flow. It might be thought that this inhibition on carotid and femoral blood flow was induced by mechanism different from that induced by the administration of pentobarbital. In order to further elucidate this question, the experiments subsequently described were performed.

E. Effect of metrazol on carotid and femoral blood flow

Effect of metrazol on carotid and femoral blood flow was examined. With the administration of metrazol, muscular discharge spontaneously emerges. Then, blood flow fluctuates in the presence of muscular discharge as mentioned by Uchiyama $^{11)}$. Therefore, there were inconveniences in measuring the blood flow 3 minutes after the administration of metrazol, since the data at that time was unstable due to the existence of muscular discharge. Therefore, the way of presentation shown in Fig. 9 was
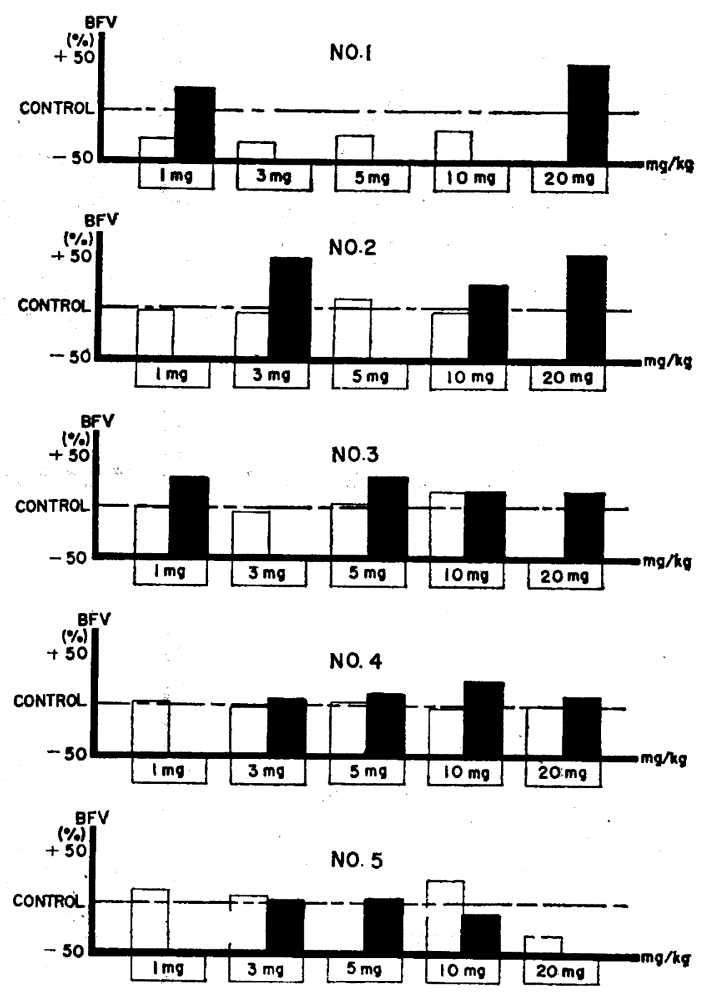

Fig. 9. Effect of metrazol on carotid blood flow. Solid bar indicates the case in which muscular discharge has been observed. Open bar indicates the case in which muscular discharge has not been currently observed. 
adopted. In this figure, the changes in carotid blood flow due to the administration of $1 \mathrm{mg} / \mathrm{kg}, 3 \mathrm{mg} / \mathrm{kg}, 5 \mathrm{mg} / \mathrm{kg}, 10 \mathrm{mg} / \mathrm{kg}$ and $20 \mathrm{mg} / \mathrm{kg}$ metrazol were shown. Solid bar indicates the case in which muscular discharge has been observed. Open bar indicates the case in which the muscular discharge has currently not been observed. Case number one points out that blood flow increased by $20 \%$, while muscular discharge has been observed, and that on the contrary, blood flow decreased by $25 \%$, while muscular discharge has currently not been observed, following the administration of $1 \mathrm{mg} / \mathrm{kg}$ metrazol. Following the administration of $20 \mathrm{mg} / \mathrm{kg}$ metrazol, muscular discharge has been observed continuously for 4 minutes, therefore there is no data available for no muscular discharge (open bar). In Fig. 10, these cases above mentioned were collected. Horizontal axis indicates the dosage of metrazol, and vertical axis indicates the ratio of variation in blood flow. With increasing dosage of metrazol, the cases of muscular discharge (solid bar) increased in number. In most of these cases, blood flow increased from $10 \%$ up to $55 \%$. On the contrary, blood flow decreased in many cases, or increased no further than $15 \%$, while muscular discharge has currently not been observed (open bar). Then the effect of metrazol on femoral blood flow was examined. The results were shown in Fig. 11 and 12 . Fig. 11

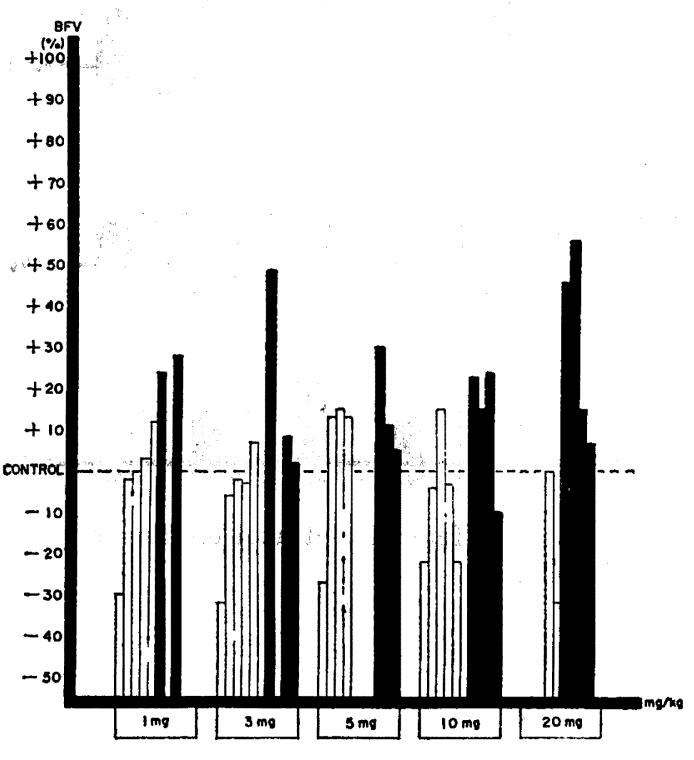

Fig. 10. Effect of metrazol on carotid blood flow. Solid bar indicates the case in which muscular discharge has been observed. Open bar indicates the case in which muscular discharge has not been currently observed.

shows individual cases, and Fig. 12 shows the amount of collection put together. In comparison of these results with those of carotid blood flow, the cases of high ratio of increment in femoral blood flow are predominant, and similarly to the data of carotid blood low, femoral blood flow increased in most cases while muscular discharge has been observed, and blood flow decreased in most cases while muscular discharge has currently not been observed. The absolute value of carotid and femoral blood flow did not always increase, even following the administration of maximum $20 \mathrm{mg} / \mathrm{kg}$ metrazol.

F. Effect of stimulation of the hippocampus on carotid and femoral blood flow

Effect of stimulation of the hippocampus, representative of archicortex, on the blood 

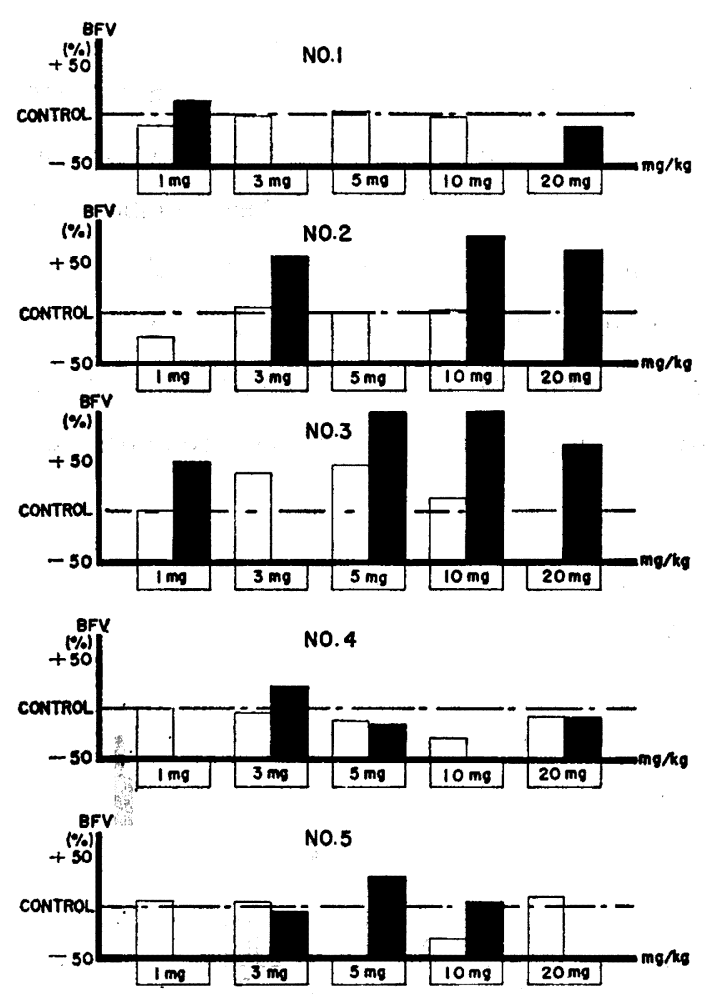

Fig. 11. Effect of metrazol on femoral blood flow.

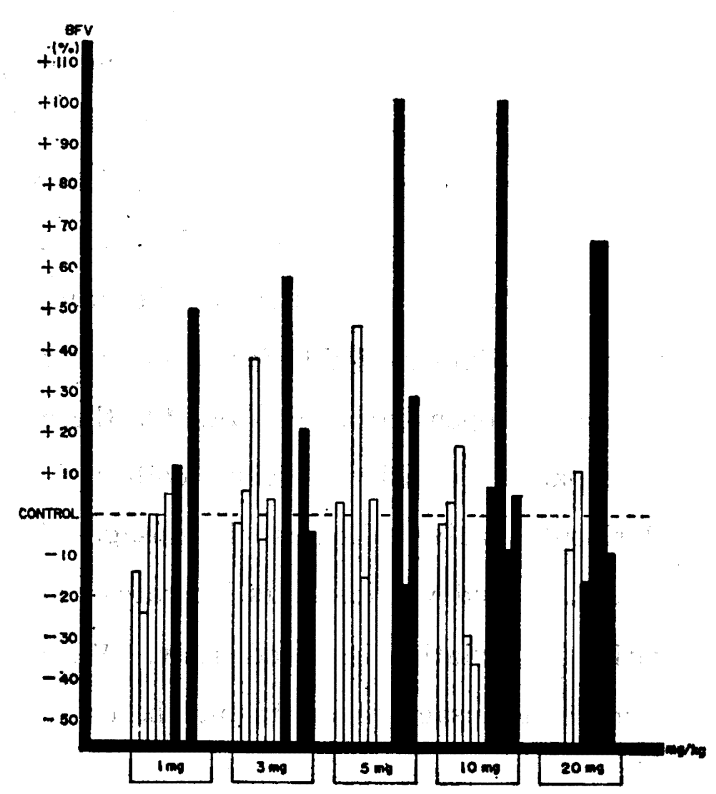

Fig. 12. Effect of metrazol on femoral blood flow.

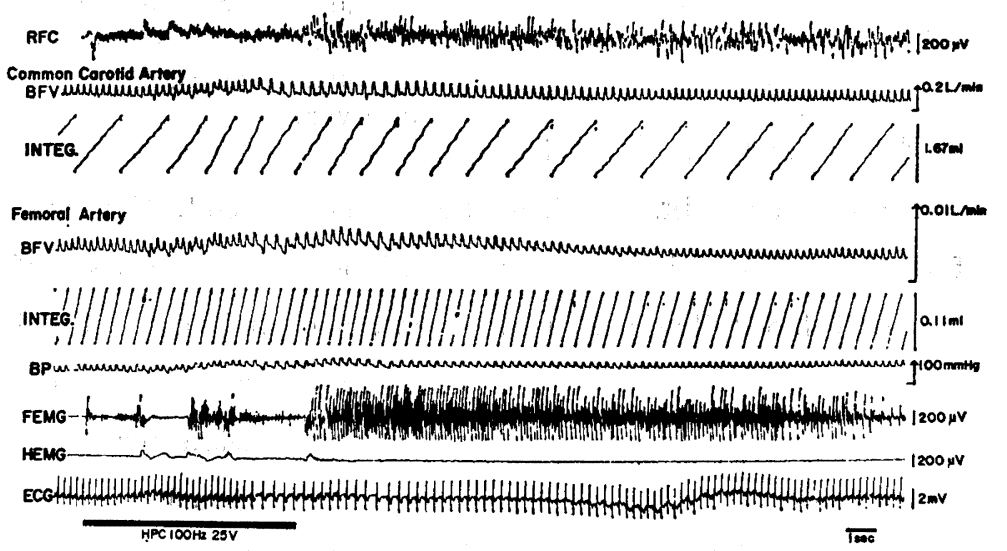

Fig. 13. Effect of stimulation of the hippocampus on EEG, carotid and femoral blood flow, arterial blood pressure, EMG and ECG. 
flow was examined. Fig. 13 shows a case of HPC stimulation at $100 \mathrm{~Hz}, 25 \mathrm{~V}$ in which both carotid and femoral blood flow began to increase 2 to 3 seconds after the beginning of stimulation and they reached the peak and regressed to the control level. These cases were collected in Fig. 14, A. Carotid blood flow (straight line) increased in 4 out of 5 cases due to HPC stimulation similar to the changes in blood flow due to MRF stimulation, but in one out of 5 cases, it did not increase. Femoral blood flow (dashed line) increased in all cases and continued to increase for over 20 seconds in 2 out of 5 cases.

G. Effect of pentobarbital on changes in blood flow due to HPC stimulation Effect of pentobarbital on changes in blood flow in response to HPC stimulation was

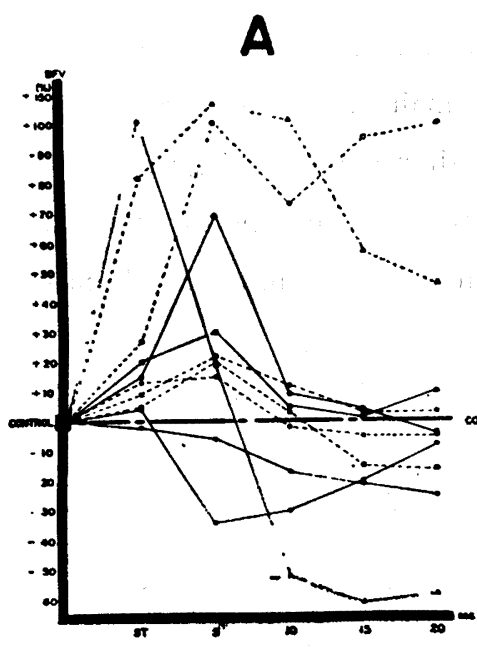

$\mathbf{B}$
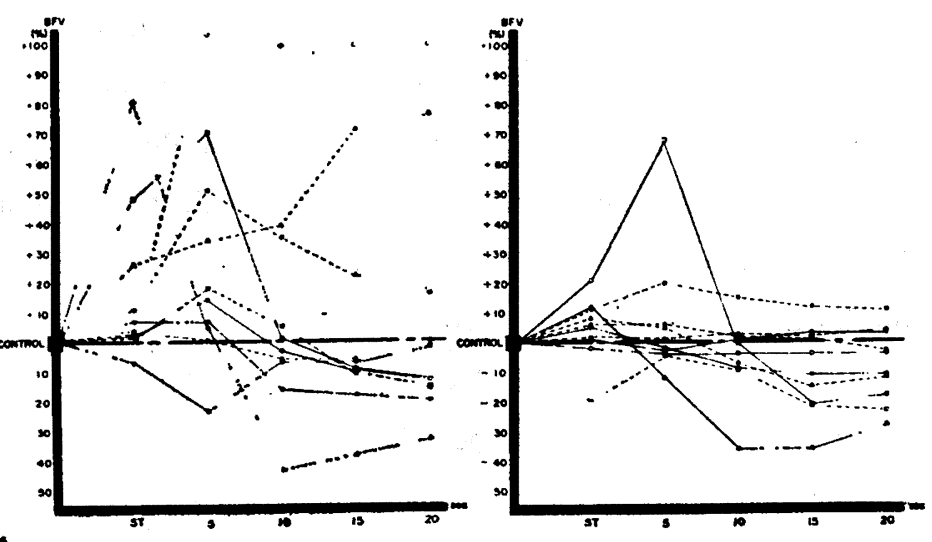

D

$\boldsymbol{E}$
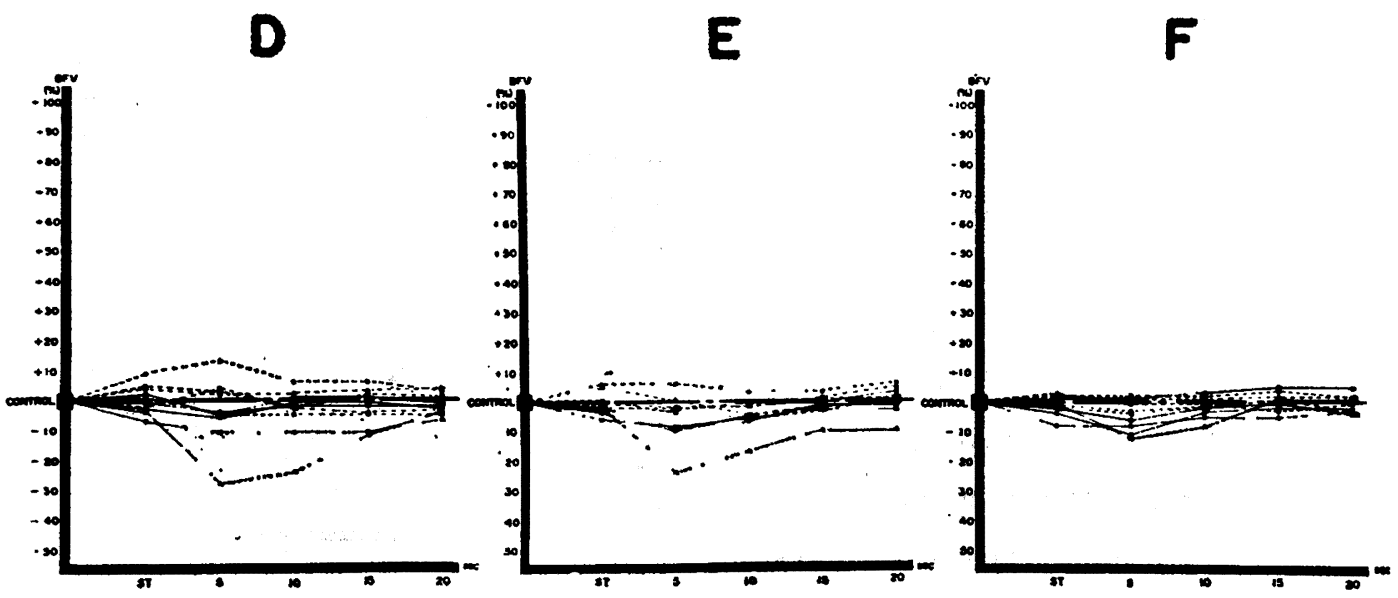

Fig. 14. Effect of pentobarbital on changes in carotid blood flow $(-)$ and femoral blood flow (......) due to stimulation of the hippocampus at $100 \mathrm{~Hz}$. 
examined. Fig. 14, $B$ indicates the changes in carotid blood flow (straight line) and femoral blood flow (dashed line) due to HPC stimulation following the administration of $1 \mathrm{mg} / \mathrm{kg}$ pentobarbital. Fig. 14, C is identical with Fig. 14, B after the administration of $3 \mathrm{mg} / \mathrm{kg}$ pentobarbital. Fig. 14, D is identical with Fig. 14, B after the administration of $5 \mathrm{mg} / \mathrm{kg}$ pentobarbital. Fig. 14, $\mathrm{E}$ is identical with Fig. 14, B after the administration of $10 \mathrm{mg} / \mathrm{kg}$ pentobarbital. Fig. 14, F is identical with Fig. 14, B after the administration of $20 \mathrm{mg} / \mathrm{kg}$ pentobarbital. Following the administration of $1 \mathrm{mg} / \mathrm{kg}$ pentobarbital as shown in Fig. 14, B, ratio of variation in femoral blood flow due to HPC stimulation declined, but slighter than that due to MRF stimulation following the administration of $1 \mathrm{mg} / \mathrm{kg}$ pentobarbital in Fig. 5, B. Following the administration of $3 \mathrm{mg} / \mathrm{kg}$ pentobarbital, the inhibitory effect was sligher than that due to MRF stimulation. Following the administration of $5 \mathrm{mg} / \mathrm{kg}$ in Fig. 14, D, $10 \mathrm{mg} / \mathrm{kg}$ in Fig. 14, E and $20 \mathrm{mg} / \mathrm{kg}$ in Fig. 14, F pentobarbital, ratio of variation in femoral blood flow remained within $10 \%$ in each case. On the other hand, increased ratio in carotid blood flow declined slightly by the administration of $1 \mathrm{mg} / \mathrm{kg}$ pentobarbital, then declined within $10 \%$ except for one case by the administration of $3 \mathrm{mg} / \mathrm{kg}$ pentobarbital, then declined within $10 \%$ in all cases by the administration of $5 \mathrm{mg} / \mathrm{kg}, 10$ $\mathrm{mg} / \mathrm{kg}$ and $20 \mathrm{mg} / \mathrm{kg}$ pentobarbital, similarly to the results of MRF stimulation after the administration of pentobarbital in Fig. 5.

H. Effect of MRF stimulation on common carotid blood flow and internal carotid blood flow

If the cervical sympathetic nerve was carefully kept intact while a probe (which requires $8 \mathrm{~mm}$ blunt dissection of the common carotid artery) was placed, there were many cases of reduction in common and internal carotid blood flow. Fig. 15 shows that MRF stimulation caused a reduction in common carotid blood flow in 12 out of 18 cases. In 6 out of 18 cases, blood flow increased. After the stimulation, in most

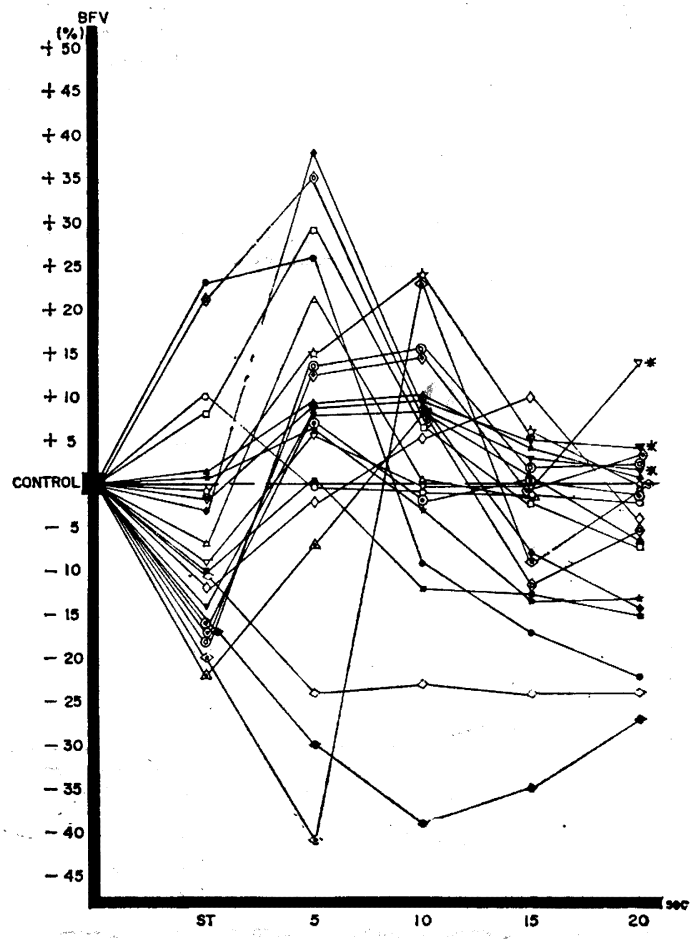

Fig. 15. Effect of MRF stimulation on common carotid blood flow and internal carotid blood flow $(*)$. 
cases, blood flow increased rapidly. This reduction in common carotid blood flow might be thought as the results of the reduction in either external carotid or internal carotid blood flow. In order to investigate whether or not the internal carotid blood flow may take part in this reduction, common carotid blood flow was measured by ligating the external carotid artery (marked $*$ in Fig. 15). Common carotid blood flow, following ligature of the external carotid artery, decreased due to MRF stimulation in all 3

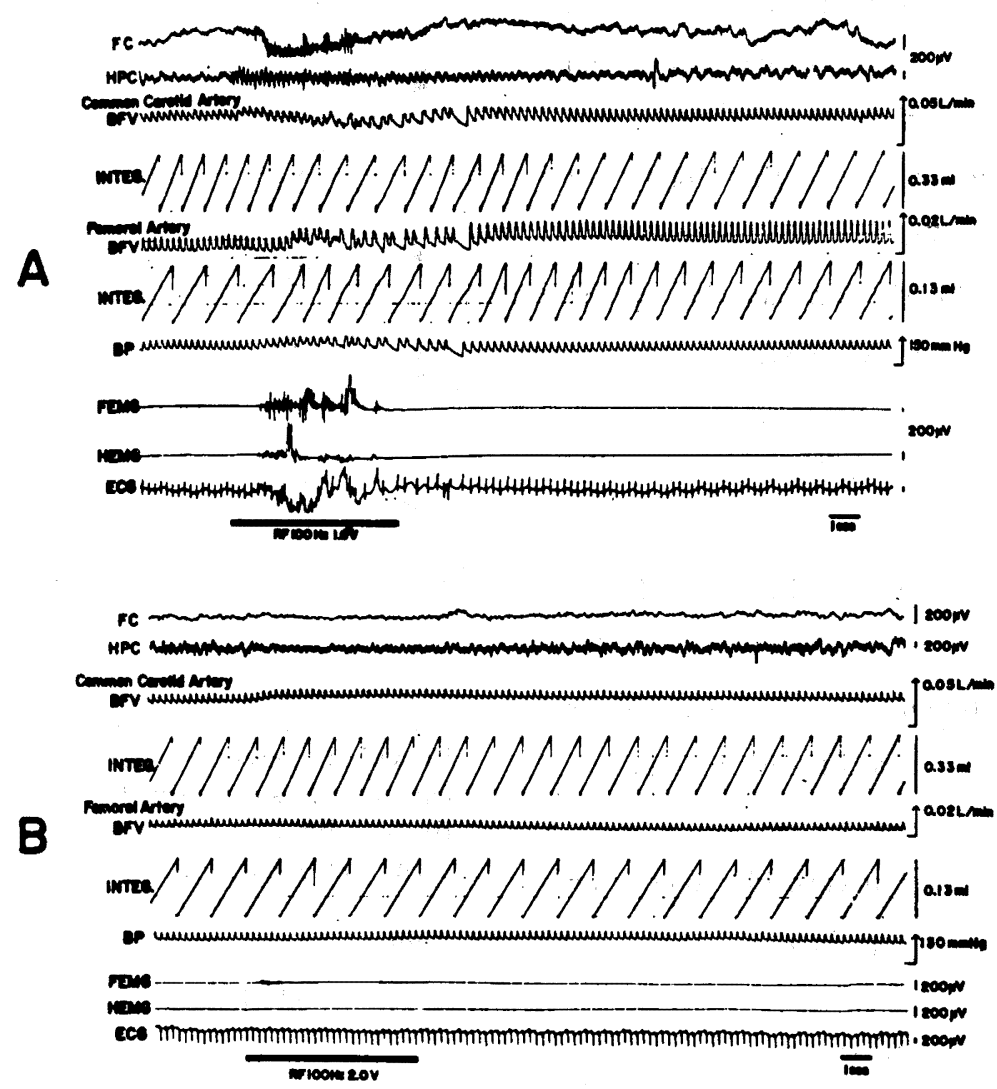

Fig. 16. Effect of MRF stimulation on EEG, carotid and femoral blood flow, arterial blood pressure, EMG and ECG. Heavy lines indicate periods of stimulation. A: A case in which theta rhythm activity in the hippocampus due to MRF stimulation was observed. B:A case in which desynchronization due to MRF stimulation was observed. Abbreviations : $\mathrm{FC}=\mathrm{EEG}$ induced from frontal cortex; HPC $=\mathrm{EEG}$ induced from the hippocampus ; $\mathrm{BFV}=$ blood flow volume ; INTEG. =integration ; $\mathrm{BP}=$ arterial blood pressure; FEMG=EMG induced from fore limb ; HEMG=EMG induced from hind limb. 
cases, and the same decreases were noticed in carotid blood flow after ligation of the internal carotid artery.

I. Carotid and femoral blood flow, arterial pressure and hippocampal EEG

In order to elucidate the cause, with keen interest in a temporary reduction in common and internal carotid blood flow, EEG of the hippocampus was added and compared with the blood flow (Fig. 16 and 17). In Fig. 16, $A$ is a case with theta rhythm activity in the hippocampus due to MRF stimulation. In Fig. 16, $B$ is a case with desynchronization. In the former, MRF stimulation at $100 \mathrm{~Hz} 1.6 \mathrm{~V}$ for 6 seconds resulted in a reduction in the carotid blood flow, in spite of the increase in arterial pressure and femoral blood flow. After the stimulation, carotid blood flow increased. In the latter, MRF stimulation caused an increase in carotid blood flow

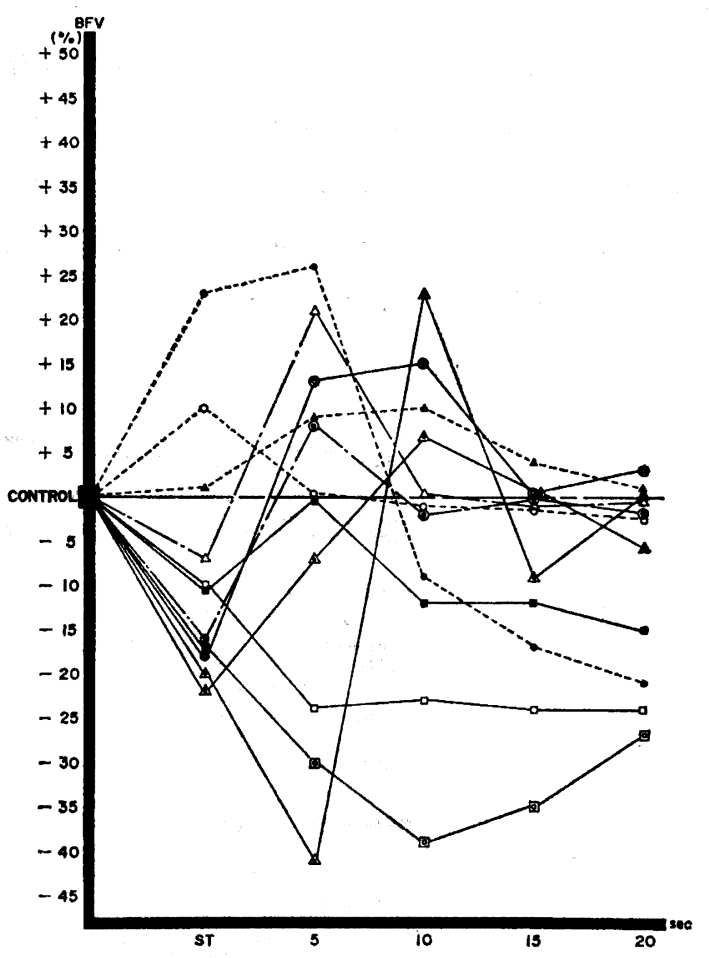

Fig. 17. Effect of MRF stimulation on carotid blood flow. Straight lines indicate cases in which theta rhythm activity in the hippocampus due to MRF stimulation was observed. Dashed lines indicate cases in which desynchronization in the hippocampus was observed. Straight-dashed lines indicate cases in which mixed pattern was observed.

and showed no change in arterial pressure and femoral blood flow. Fig. 17 shows 11 cases that were collected from these experiments. Dashed lines indicate the cases with desynchronization in the hippocampus due to MRF stimulation. Straight lines indicate the cases with theta rhythm activity in the hippocampus due to MRF stimulation. There were 3 cases with blood flow increase and 8 cases with blood flow decrease due to MRF stimulation. All cases with increased blood flow displayed desynchronization in the hippocampus, while 6 out of 8 cases with decreased blood flow displayed theta rhythm activity and 2 out of 8 cases displayed mixed pattern. During the presence of hippocampal theta rhythm activity, arterial pressure increased and heart rate decreased. During the presence of desynchronization, no changes were noticed in either arterial pressure or heart rate. The relations between femoral blood flow and hippocampal EEG were as follows. 4 out of 9 cases were with theta rhythm activity and in all of 
them, femoral blood flow increased while carotid blood flow decreased. 3 out of 9 cases were with desynchronization, and in the first one of the 3 , femoral blood flow increased and carotid blood flow increased, in the second one, femoral blood flow decreased while carotid blood flow increased and in the third one, femoral blood flow showed no change whereas carotid blood flow increased. 2 out of 9 cases were with mixed pattern and in the first one of the 2, femoral and carotid blood flow decreased, in the second one, femoral increased while carotid blood flow decreased. Relations between carotid and femoral blood flow were as follows: 6 cases with carotid blood flow decrease while femoral blood flow increased; 2 cases with carotid blood flow increase while femoral blood flow decreased; 2 cases with both carotid and femoral blood flow increase; one case with both carotid and femoral blood flow decrease. Then, blood flow of the vertebral artery, through which the brain is irrigated besides the carotid artery, was measured in the same experiments and the relations between vertebral blood flow and hippocampal EEG were investigated.

J. Effect of MRF stimulation on ver-

tebral blood flow and hippocampal EEG

Straight, dashed and straight-dashed lines in Fig. 18 indicate respectively the cases with theta rhythm activity, desynchronization and mixed pattern similar to Fig. 17. Three out of 5 cases with blood flow increase due to MRF stimulation displayed desynchronization, the fourth one a mixed pattern and the fifth one a theta rhythm activity. All cases with decreased blood flow displayed theta rhythm activity. After the stimulation, blood flow increased rapidly in all cases. In comparison with the changes in vertebral blood flow in Fig. 18 and in carotid blood flow in Fig. 17, it is understood that the increased ratio of vertebral blood flow during stimulation is greater than that of

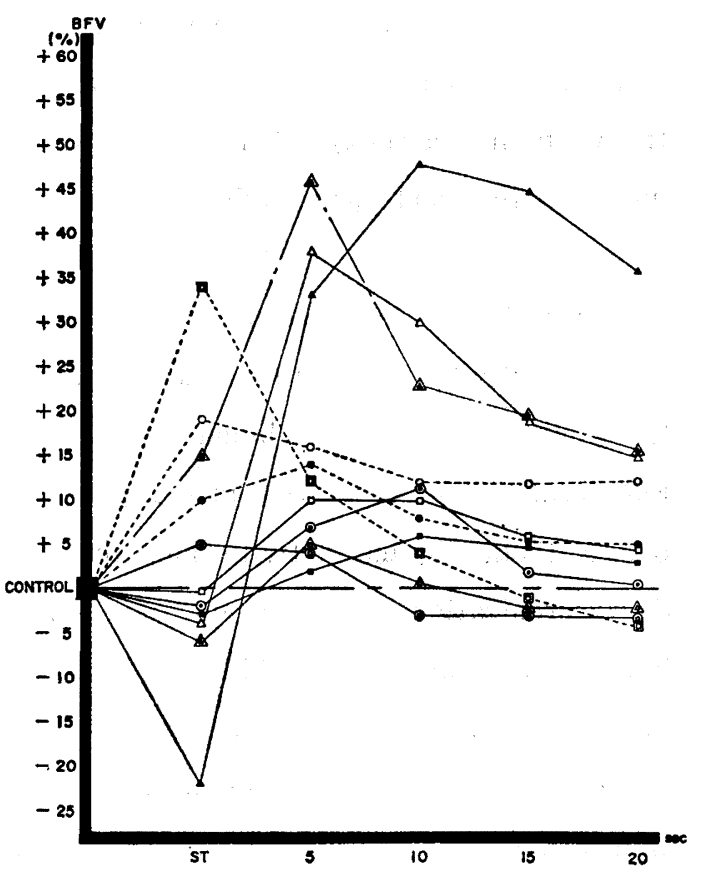

Fig. 18. Effect of MRF stimulation on vertebral blood flow. Straight lines indicate cases in which theta rhythm activity was observed. Dashed lines indicate cases in which desynchronization was observed. Straight-dashed line indicates a case in which mixed pattern was observed. 
carotid blood flow, that the decreased ratio in vertebral blood flow during stimulation is smaller than that in carotid blood flow and that there are close relations between theta rhythm activity and decreased vertebral blood flow, and those between desynchronization and increased vertebral blood flow, similar to the relations between hippocampal EEG and carotid blood flow. But there are some exceptions.

K. Effect of MRF stimulation on regional cortical blood flow (rCoBF)

Thus, as above mentioned, the relations between hippocampal EEG and both carotid and vertebral blood flow were examined. Then those between hippocampal EEG and regional cortical blood flow were examined. The results were shown in Fig. 19. Arrows extending upwards indicate blood flow increase, arrows extending sideways indicate the cases which show no change in blood flow, and arrows extending downwards indicate blood flow decrease. Inclination of arrows indicates the grade of either increase or decrease. Changes in $\mathrm{rCoBF}$, during and after the stimulation, are displayed respectively by means of arrows. The patterns of hippocampal EEG are displayed in the column of HPC EEG. Solid circles indicate the cases with theta rhythm activity, half solid circles indicate mixed pattern and open circles indicate desynchronization. Three out of 4 cases with blood flow decrease during MRF stimulation displayed a theta rhythm activity, the other one a mixed pattern. Two out of 4 cases with blood flow increase during MRF stimulation displayed mixed pattern, the third one a theta rhythm activity and the fourth one a desynchronization. Three out of 5 cases with no blood flow change during MRF stimulation displayed mixed pattern, the fourth one a theta rhythm activity and the fifth one a desynchronization. Comparing these results with those of common and vertebral blood flow, there are correlations between theta rhythm activity and decreased blood flow similar to the correlations between theta rhythm activity and both common and vertebral blood flow. On the contrary, there is less correlation between increased

\begin{tabular}{|c|c|c|c|}
\hline \multirow{2}{*}{ NO } & \multirow{2}{*}{$\begin{array}{l}\text { HPC } \\
\text { EEG }\end{array}$} & \multicolumn{2}{|c|}{ RF STIM } \\
\cline { 3 - 4 } & during & ofter \\
\hline 1 & $\bullet$ & $\rightarrow$ & $\uparrow$ \\
\hline 2 & $\bullet$ & $\nearrow$ & $\uparrow$ \\
\hline 3 & $\bullet$ & $\rightarrow$ & $\uparrow$ \\
\hline 4 & 0 & $\rightarrow$ & $\uparrow$ \\
\hline 5 & 0 & $\rightarrow$ & $\uparrow$ \\
\hline 6 & 0 & $\rightarrow$ & $\uparrow$ \\
\hline 7 & 0 & $\rightarrow$ & $\uparrow$ \\
\hline 8 & 0 & $\rightarrow$ & $\uparrow$ \\
\hline 9 & 0 & $\rightarrow$ & $\uparrow$ \\
\hline 10 & 0 & $\rightarrow$ & $\uparrow$ \\
\hline 11 & 0 & $\rightarrow$ & $\downarrow \uparrow$ \\
\hline 12 & 0 & $\rightarrow$ & $\uparrow$ \\
\hline 13 & 0 & $\nearrow$ & $\uparrow$ \\
\hline
\end{tabular}

Fig. 19. Effect of MRF stimulation on regional cortical blood flow.

: Cases in which theta rhythm activity in the hippocampus was observed. $O:$ Cases in which desynchronization was observed. D: Cases in which mixed pattern was observed. 
$\mathrm{r}-\mathrm{CoBF}$ and desynchronization than that between both increased common and vertebral blood flow and desynchronization. The latency of changes in $r-C o B F$ due to MRF stimulation is approximately 2 seconds, and it is longer than that of common and vertebral blood flow. After the stimulation, in 12 out of 13 cases, r-CoBF increased rapidly. The other one showed decrease and reached the peak 2 seconds after stimulation, then turned into increase and reached the peak 18 seconds after stimulation. Then, the sites of stimulation in the reticular formation, which caused different patterns in the hippocampus, were histologically examined, but the histological difference was not found.

L. Effect of MRF stimulation on EEG and carotid blood flow under various intracranial pressures

Following the elevation of intracranial pressure, the results shown in Fig. 20 and 21

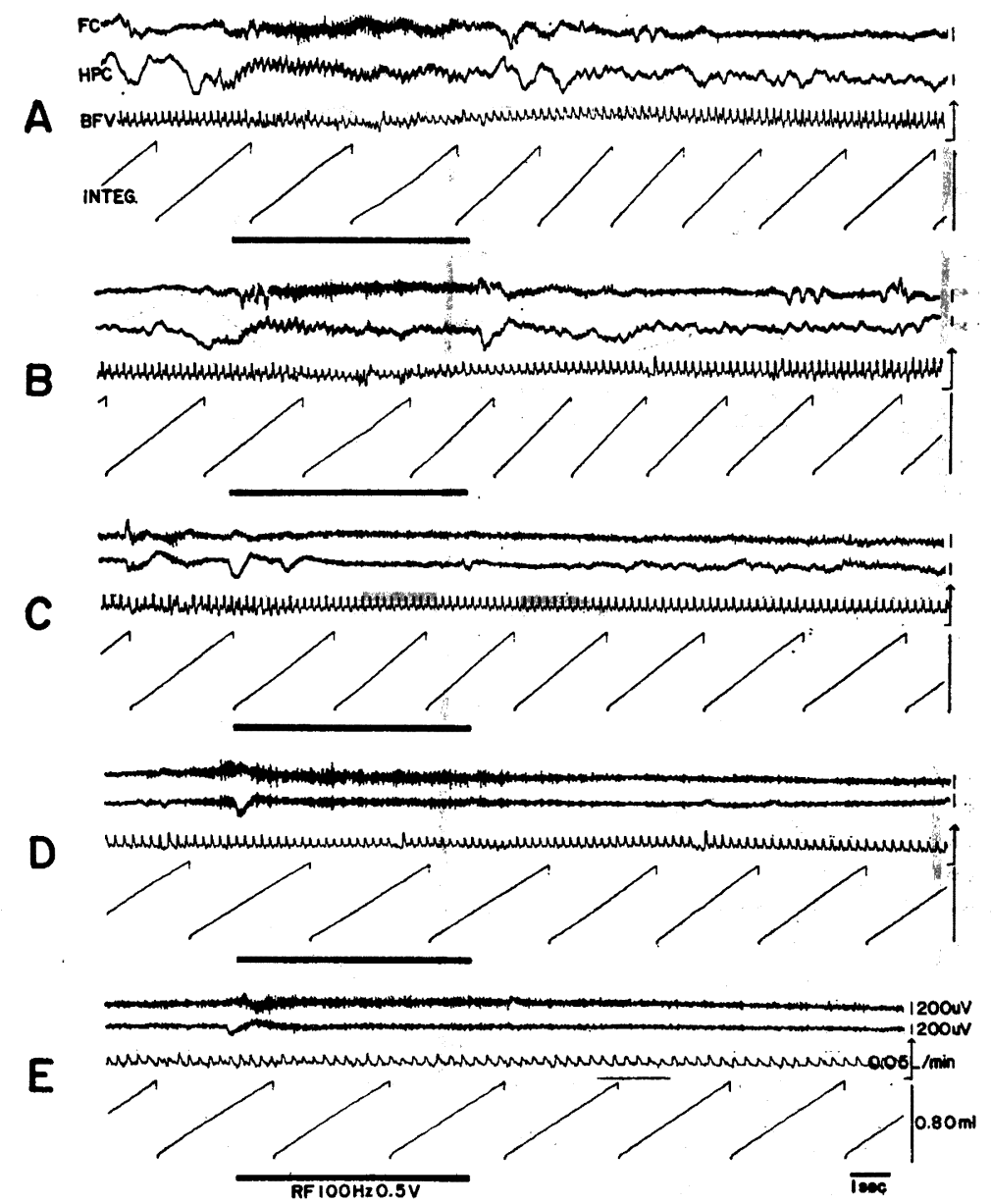

Fig. 20. Effect of MRF stimulation on EEG and carotid blood flow under various intracranial pressures. 
$S-30$

were obtained. Fig. 20, $\mathbf{A}$ indicates changes in EEG and carotid blood flow due to MRF stimulation under normal ICP. Fig. 20, B indicates changes in EEG and carotid blood flow due to MRF stimulation under ICP of $30 \mathrm{mmHg}$. Fig. 20, C indicates changes in EEG and carotid blood flow under ICP of $50 \mathrm{mmHg}$. Fig. 20,D indicates changes in EEG and carotid blood flow under ICP of $70 \mathrm{mmHg}$. Fig. 20, $\mathbf{E}$ indicates changes in

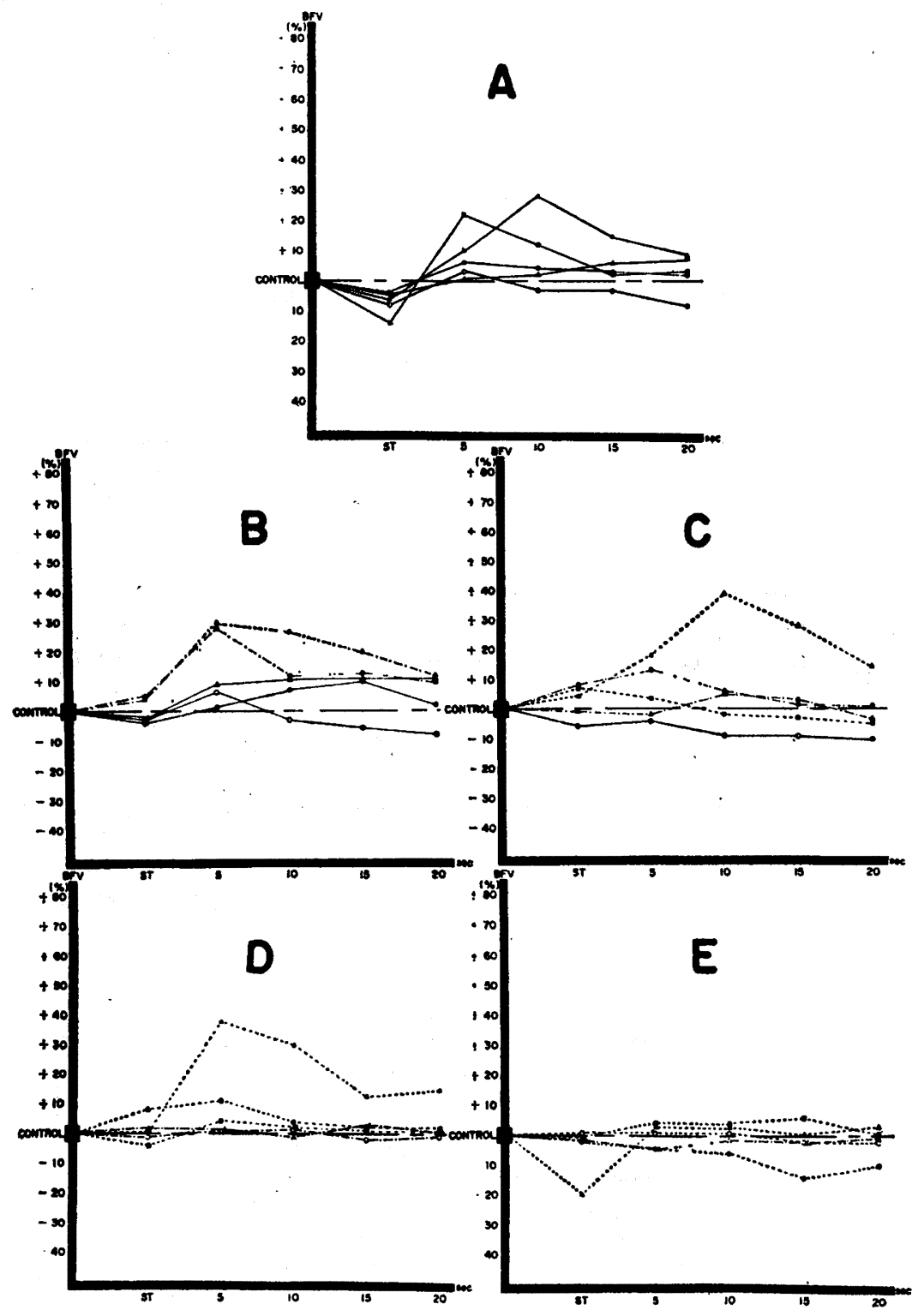

Fig. 21. Graph of changes in carotid blood flow due to MRF stimulation under various intracranial pressures. Cases with theta rhythm activity. ….. : Cases with desynchronization. -- Cases with mixed pattern. 
EEG and carotid blood flow under ICP of $90 \mathrm{mmHg}$. Under normal ICP in Fig. 20, A, carotid blood flow decreased temporarily and hippocampal EEG became synchronized in response to MRF stimulation. Then, hippocampal EEG came to show mixed pattern and temporary reduction in carotid blood flow became slighter when ICP increased up to 30 mmHg. When ICP increased up to $50 \mathrm{mmHg}$, hippocampal EEG became desynchronized and carotid blood flow turned itself into increase due to MRF stimulation, and finally blood flow stopped changing at $70 \mathrm{mmHg}$. Fig. 21 shows a collection of these experiments. Fig. 21, A indicates changes in EEG and carotid blood flow under normal ICP. Fig. 21, B indicates changes in EEG and carotid blood flow under ICP of $30 \mathrm{mmHg}$. Fig. 21, C indicates changes in EEG and carotid blood flow under ICP of $50 \mathrm{mmHg}$. Fig. 21, D indicates changes in EEG and carotid blood flow under ICP of $70 \mathrm{mmHg}$. Fig. 20, $\mathbf{E}$ indicates changes in EEG and carotid blood flow under ICP of $90 \mathrm{mmHg}$. During the stimulation of MRF, carotid blood flow decreased and synchronization was observed in the hippocampus in all cases of control : normal ICP. With the increase of ICP (30 $\mathrm{mmHg}, 50 \mathrm{mmHg}$ ), the cases with mixed pattern and desynchronization in the hippocampus during the stimulation of MRF increased in number. In these cases, carotid blood flow increased during the stimulation of MRF. When ICP increased up to 70 and $90 \mathrm{mmHg}$, EEG pattern of the hippocampus became desynchronized in most cases, and blood flow stopped changing in spite of MRF stimulation. It was postulated from these results that there are close relations between ICP and the hippocampal EEG, and between the hippocampal EEG and carotid blood flow (i. e. synchronization vs. blood flow decrease, desynchronization vs. blood flow increase.)

Then, in order to elucidate the mechanism of the hippocampal desynchronization, experiments of stimulation of the lateral hypothalamus ( $\mathrm{LH})$ were performed. Stimulation of the lateral hypothalamus, however, did not readily produce the hippocampal desynchronization. On the contrary, stimulation of the medial hypothalamus $(\mathrm{MH})$ readily produced the theta rhythm activity. As to the patterns of hippocampal EEG, strength of MRF stimulation may affect them to some extent. Namely, there were some cases in which theta rhythm activity due to MRF stimulation at $1 \mathrm{~V}$ changed into desynchronization with the increase in the strength of stimulation, and desynchronization at 1 to $2 \mathrm{~V}$ changed into theta rhythm activity with the increase in the strength.

M. Effect of cervical sympathetic nerve cut on changes in carotid blood flow due to MRF stimulation

To understand how the carotid blood flow changes in response to MRF stimulation, following experiments were performed. Fig. 22, A indicates changes in carotid blood 


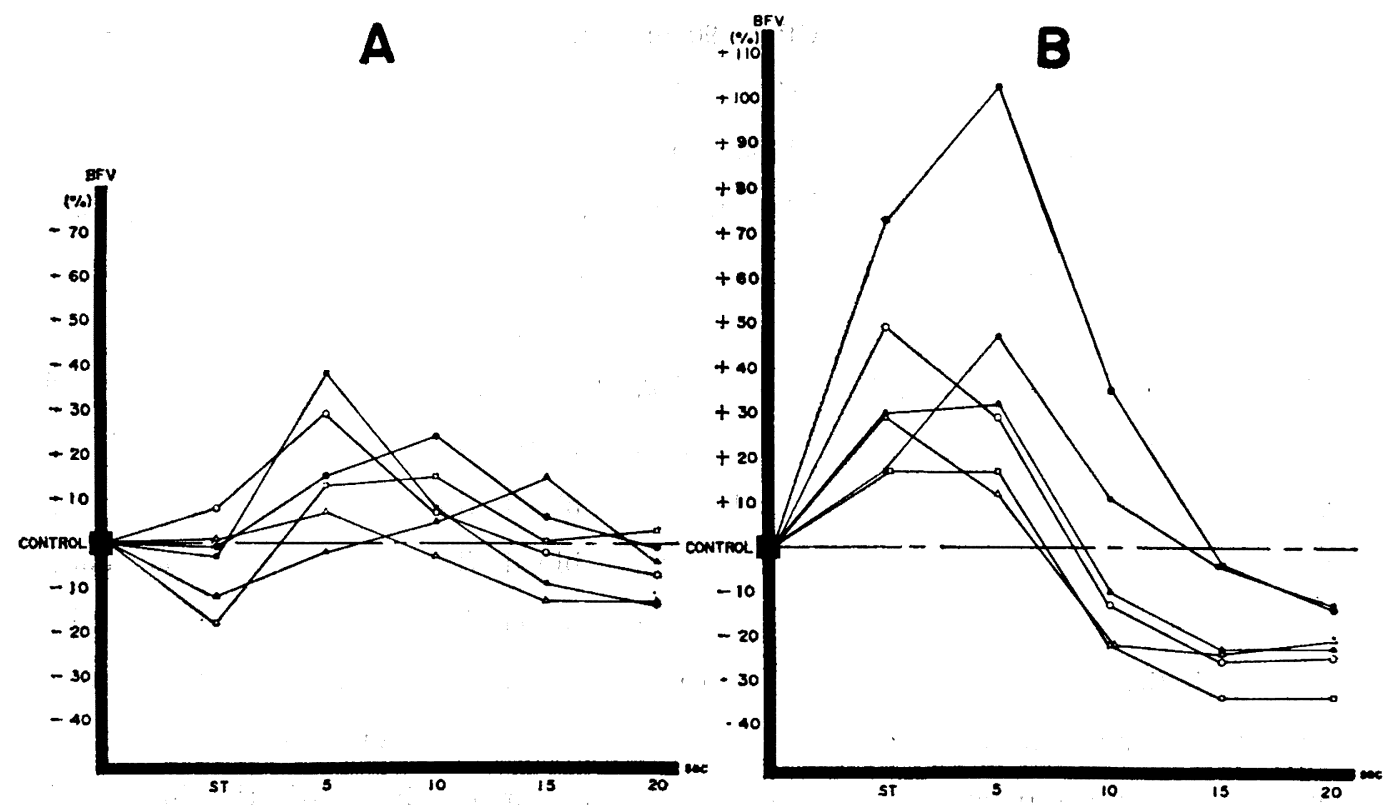

Fig. 22. Effect of cervical sympathetic nerve cut on changes in carotid blood flow in response to MRF stimulation. A : Before the nerve was cut off. B:After the nerve was cut off.

flow due to MRF stimulation before the cervical sympathetic nerve was cut off, and so does Fig. 22, B after it was cut off. Carotid blood flow decreased in 3 out of 6 cases and increased in one out of 6 cases during MRF stimulation in Fig. 22, A. The ratio of variation of blood flow was from plus $10 \%$ to minus $18 \%$. After the cut of the cervical sympathetic nerve, carotid blood flow increased in all cases during MRF stimulation in Fig. 22, B. The increased ratio was plus $73 \%$ down to plus $18 \%$. The absolute value of carotid blood flow also increased from plus $50 \%$ down to plus $13 \%$, with the average of $24 \%$. It was understood from these results that the cervical sympathetic nerve has a relation with reduction of carotid blood flow during MRF stimulation. Slight decrease or no change in $\mathrm{r}-\mathrm{CoBF}$ due to MRF stimulation changed into rapid increase by the cervical sympathetic nerve cut. Then, in order to elucidate the mechanism of increase in carotid blood flow due to MRF stimulation after the nerve was cut off, cholinergic tracts in the brain (the septal-hippocampal pathway and habenulo-interpeduncular pathway) were examined. Impairment of either septum or fimbria which belongs to former tract showed no significant changes. Following impairment of the interpeduncular nucleus, results subsequently described, were obtained.

N. Effect of IPN lesion on changes in carotid blood flow due to MRF stimulation with the cervical sympathetic nerve cut off 

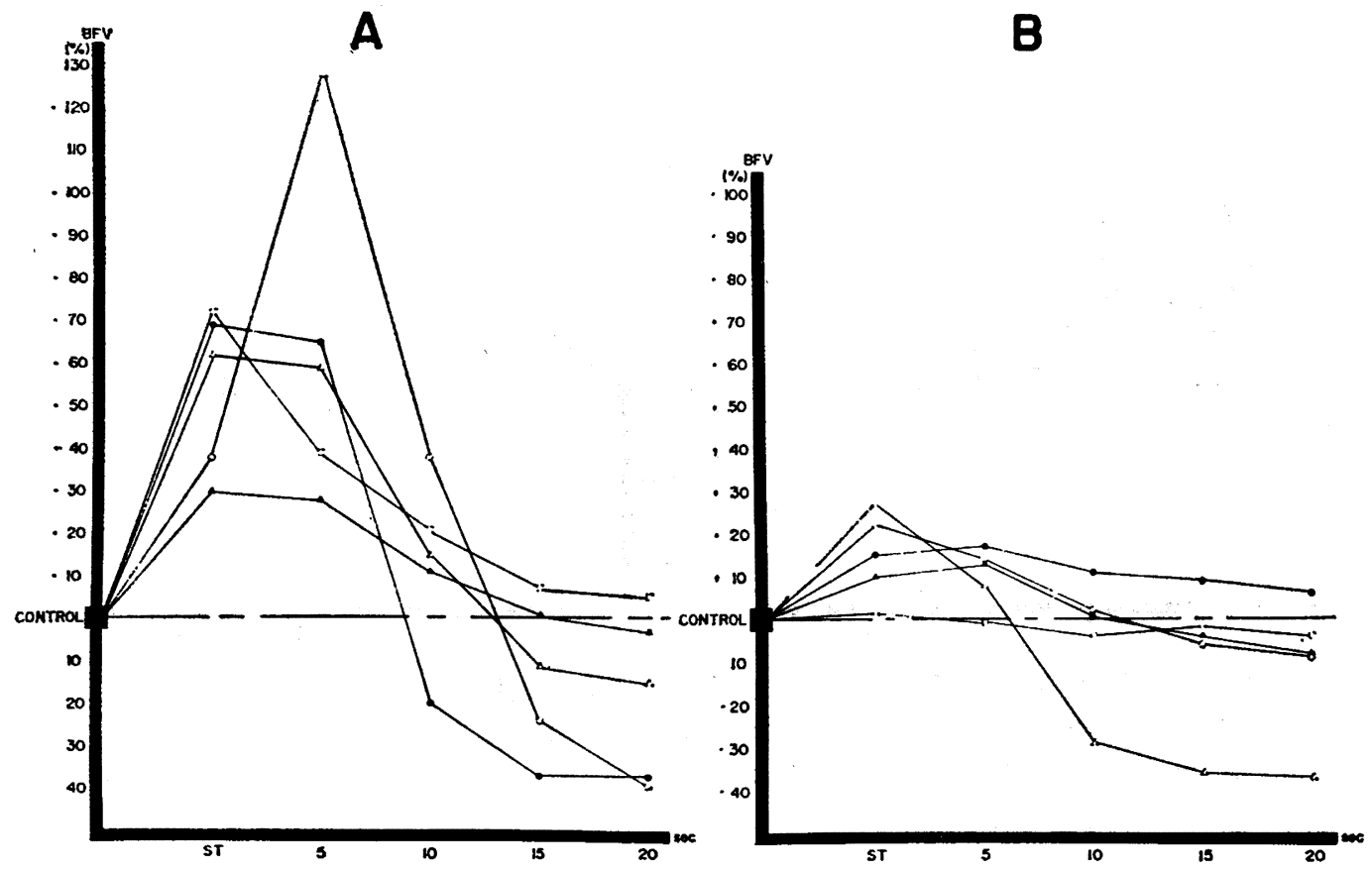

Fig. 23. Effect of IPN impairment on changes in carotid blood flow due to MRF stimulation with the cervical sympathetic nerve cut off. A : Before the impairment of IPN. B : After the impairment of IPN.

30 minutes after the impairment of IPN which contains a great amount of choline acetyltransferase, MRF stimulation was performed. Fig. 23, A shows control, and Fig. 23, B shows the changes after the impairment of IPN. The increased ratio of blood flow decreased below $30 \%$ by impairment of IPN. The same result was confirmed in $\mathrm{r}-\mathrm{CoBF}$.

O. Effect of atropine on changes in carotid blood flow due to MRF stimulation with the cervical sympathetic nerve cut off

Almost the same results were obtained following the administration of atropine. Carotid blood flow increased from $10 \%$ up to $60 \%$ due to MRF stimulation before the administration of atropine, in Fig. 24, A. Following the administration of $0.3 \mathrm{mg} / \mathrm{kg}$ atropine, this increased ratio of the blood flow declined to distribute from $3 \%$ to $21 \%$, in Fig. 24, B. From the results of the impairment of IPN and administration of atropine, it might be thought that IPN, considered to belong to cholinergic tract, has something to do with neurogenic control of the blood flow. In order to examine this from a different point of view, experiments of IPN stimulation were performed.

P. Effect of IPN stimulation on carotid blood flow 

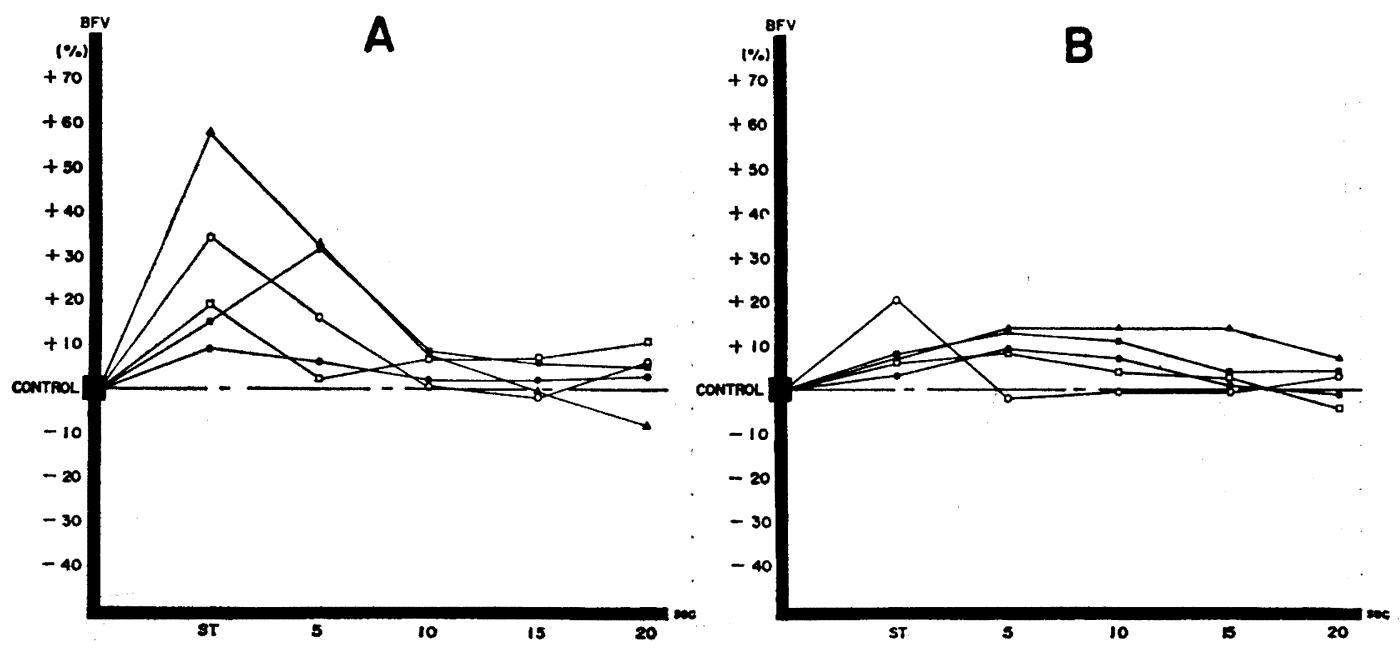

Fig. 24. Effect of atropine on changes in carotid blood flow due to MRF stimulation with the cervical sympathetic nerve cut off.

A : Before the administration of atropine. B : After the administration of atropine $(0.3 \mathrm{mg} / \mathrm{kg})$.

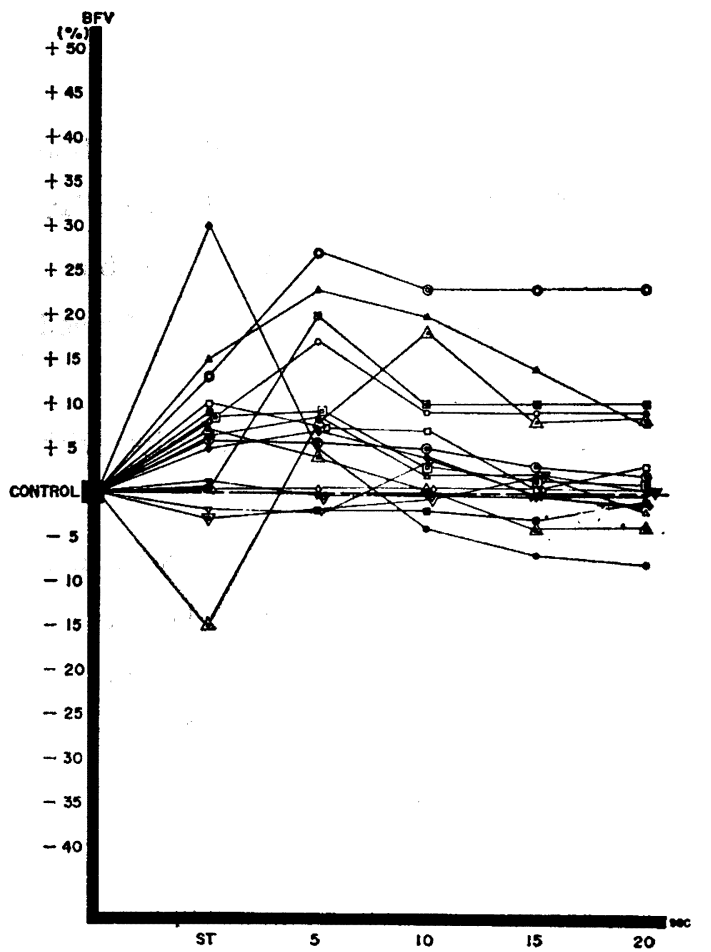

Fig. 25. Effect of IPN stimulation on carotid blood flow.
Table. 1. Effect of MRF stimulation on arterial blood gases and pH. Arterial samples were taken during stimulation, note: (During), 5 seconds after the end of stimulation, note : (5-10 sec after) and 20 seconds after the end of stimulation, note : (20-25 sec after).

\begin{tabular}{|c|c|c|c|c|c|}
\hline No & Blood Gases & Control & $\begin{array}{l}\text { During } \\
(6 \circ c)\end{array}$ & $\begin{array}{l}5-10 \text { sec } \\
\text { ofter }\end{array}$ & $\begin{array}{c}20-25 s e c \\
\text { affer }\end{array}$ \\
\hline \multirow{4}{*}{1} & $\mathrm{pH}$ & 7.322 & 7.354 & 7.386 & 7.331 \\
\hline & $\mathrm{PaO2}$ & 92.0 & 93.9 & 97.4 & 93.3 \\
\hline & Paco2 & 30.5 & 27.9 & 26.7 & 28.9 \\
\hline & $\mathrm{HCO}_{3}^{-}$ & 15.4 & 15.7 & 19.1 & 15.5 \\
\hline & $\mathrm{CO}_{2}$ Content & 16.5 & 16.7 & 20.1 & 16.5 \\
\hline & Soor & 95 & 96 & 97 & 95.5 \\
\hline \multirow{6}{*}{2} & $\mathrm{pH}$ & 7.384 & 7.398 & 7.349 & 7.379 \\
\hline & Paor & 107.9 & 108.6 & 110.1 & 108.2 \\
\hline & Paco2 & 25.4 & 23.0 & 29.9 & 24.0 \\
\hline & $\mathrm{HCO}_{3}^{-}$ & 15.1 & 14.3 & 15.9 & 14.5 \\
\hline & $\mathrm{CO}_{2}$ Content & 16.2 & 15.4 & 17.2 & 15.6 \\
\hline & $\mathrm{SaO2}$ & 98 & 98 & 97.5 & 98 \\
\hline \multirow{6}{*}{3} & pH & 7.495 & 7.518 & 7.507 & 7.518 \\
\hline & Paoz & 91.6 & 94.4 & 95.3 & 88.7 \\
\hline & $P a c 02$ & 29.5 & 25.4 & 27,6 & 24.7 \\
\hline & $\mathrm{CO}_{2}$ Content & 22.3 & 20.3 & 21.5 & 19.6 \\
\hline & $\mathrm{HCOs}^{-}$ & 23.2 & 21.1 & 22.4 & 20.4 \\
\hline & $\mathrm{SOO2}$ & 97.5 & 98 & 98 & 98 \\
\hline
\end{tabular}

PaO2, Poco2 (mmHg) HCOs, CO2 Content (mEq/L) SoO2 (\%) 
In 11 out of 17 cases, carotid blood flow increased by $5 \%$ up to $30 \%$ due to IPN stimulation at $100 \mathrm{~Hz} 0.4$ to $2.0 \mathrm{~V}$, in Fig. 25. In most cases, the increased ratio was less than $10 \%$. In 3 out of 17 cases, blood flow decreased. From the results of experiments of the cervical sympathetic nerve cut, impairment of IPN, administration of atropine and stimulation of IPN, it is postulated that the early change in carotid blood flow due to MRF stimulation is regulated by the nerve. It is not known whether the early changes in blood flow, starting 2 to 3 seconds after the beginning of stimulation, are regulated only by the nerve or by both metabolic products and the nerve. Then, in order to clarify this problem, blood gases were measured.

Q. Effect of MRF stimulation on arterial blood gases and $\mathrm{pH}$

Effect of MRF stimulation on arterial blood gases and $\mathrm{pH}$ was examined, and the results were shown in Table 1. There are only three cases, but in all cases, changes in blood gases and $\mathrm{pH}$ were found during stimulation. $\mathrm{PaO}_{2}$ increased, $\mathrm{PaCO}_{2}$ decreased

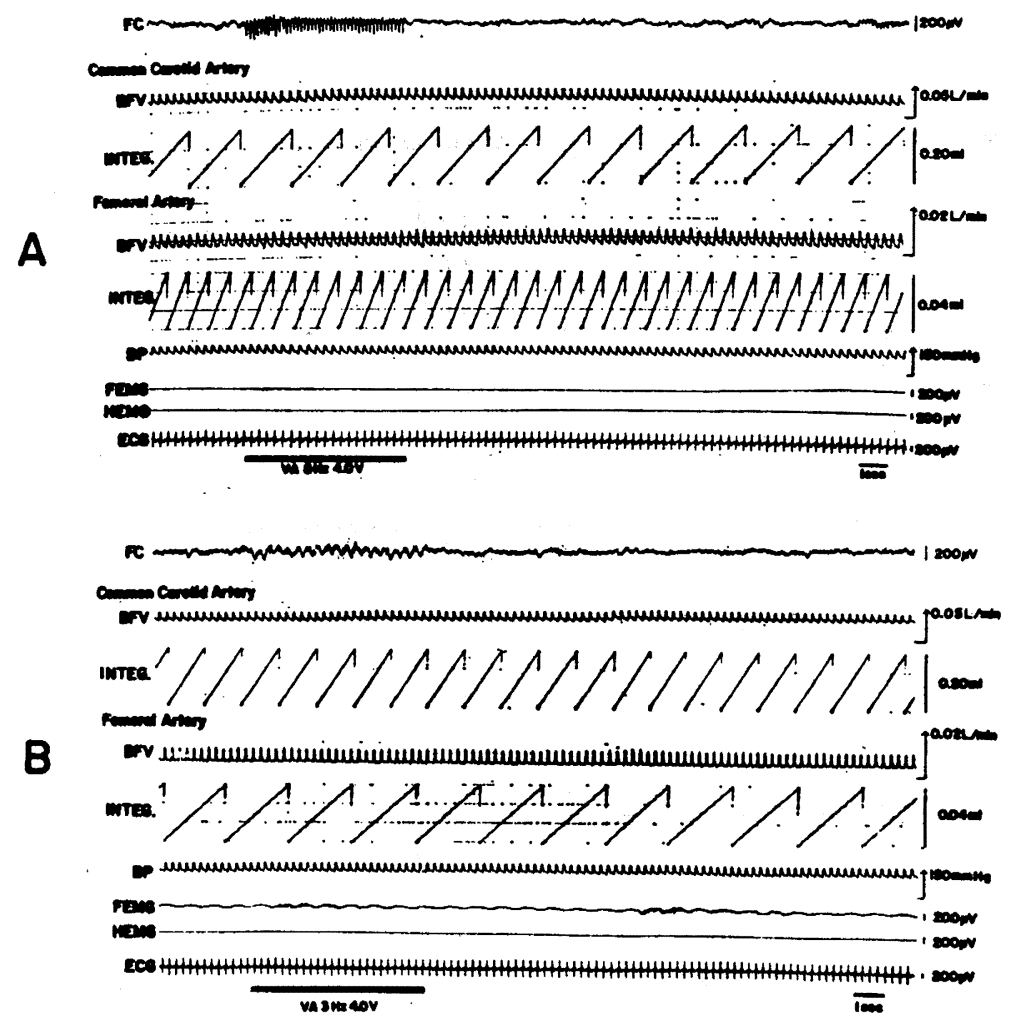

Fig. 26. Effect of VA stimulation on EEG, carotid and femoral blood flow, arterial blood pressure, EMG and ECG. A : VA stimulation at $8 \mathrm{~Hz}$. B :VA stimulation at $3 \mathrm{~Hz}$. 
and $\mathrm{pH}$ increased. 5 to 10 seconds after stimulation, they kept changing considerably, 20 to 25 seconds after stimulation, they began to regress to the control level.

R. Effect of VA stimulation on carotid and femoral blood flow

It is well noted that VA stimulation at $8 \mathrm{~Hz}$ produces a recruiting response, and that the diffuse thalamo cortical projection system, which has a relation to this response, is thought to be the inhibitory system in the brain. It is also noted that VA stimulation at $3 \mathrm{~Hz}$ produces spike and wave. In this chapter, the cardiovascular changes during the presense of these responses were examined. Fig. 26, A shows data of carotid and femoral blood flow, arterial blood pressure, EMG and ECG during the presence of recruiting response due to $\mathrm{VA}$ stimulation at $8 \mathrm{~Hz}$. No significant changes were found in this figure. Fig. 26, B shows data during the presence of spike and wave due to VA stimulation at $3 \mathrm{~Hz}$. No significant changes were found. Fig. 27 shows 6 cases that were collected from these experiments. In 2 out of 6 cases, carotid blood flow decreased approximately by $10 \%$ during the presence of recruiting response, but neither

A
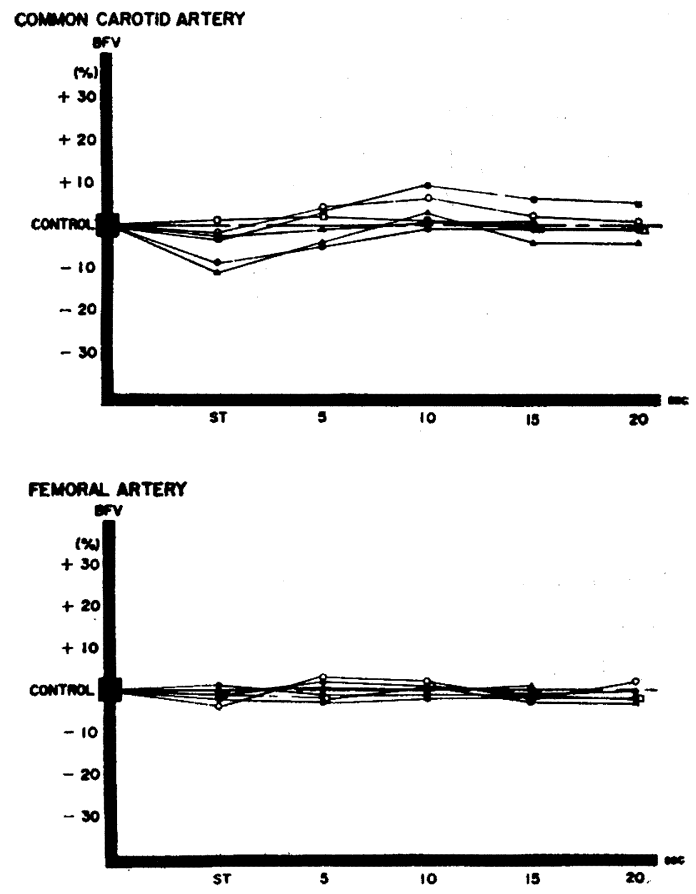

B
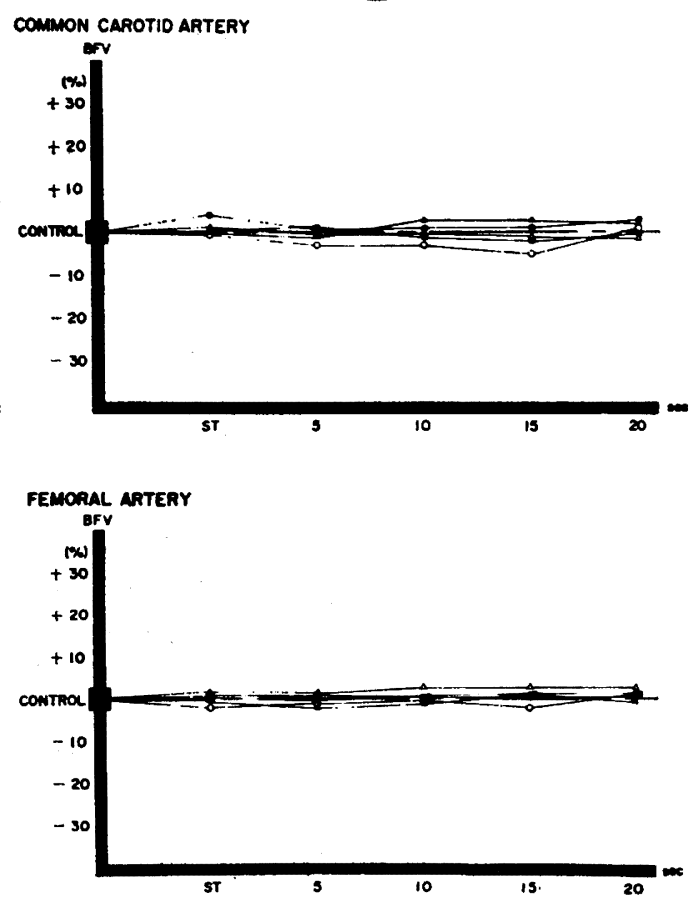

Fig. 27. Effect of VA stimulation on carotid and femoral blood flow. $A$ : With VA stimulation at $8 \mathrm{~Hz}$, recruiting response has been observed in the cerebral cortex. B:With VA stimulation at $3 \mathrm{~Hz}$, spike and wave has been observed. 


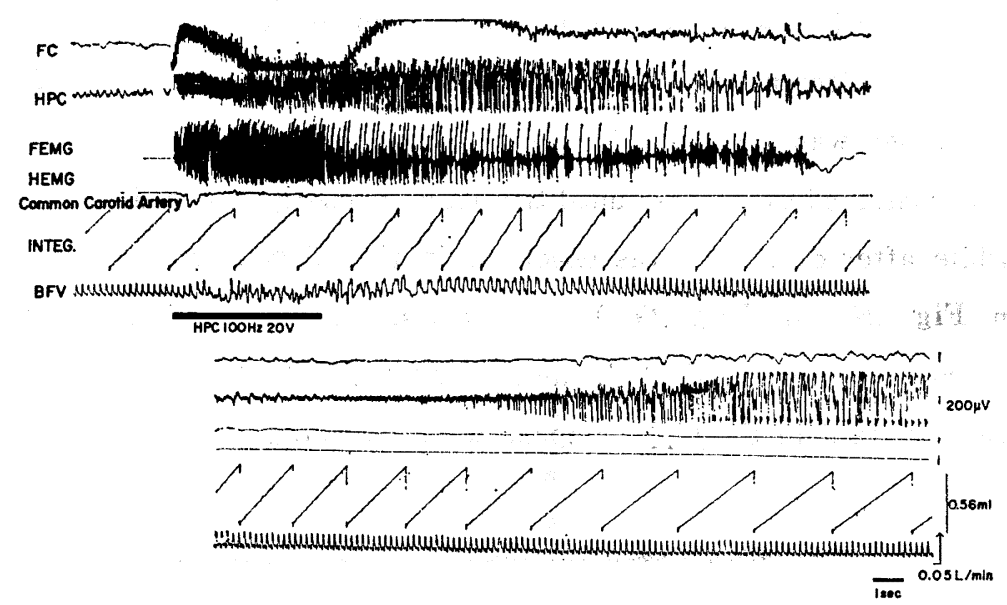

Fig. 28. Effect of stimulation of the hippocampus on EEG,

EMG and carotid blood flow.

femoral blood flow nor arterial blood pressure changed during that time. No significant changes were found in cardiovascular system during the presence of spike and wave.

S. Effect of HPC stimulation on carotid blood flow

Carotid blood flow did not change due to weak stimulation of the hippocampus which did not produce the muscular discharge. This result differs from that of MRF stimulation. The changes in carotid blood flow, however, were similar to the results of MRF stimulation during the presence of muscular discharge. That is to say, the temporal reduction during stimulation were followed by the rapid increase after stimulation in most cases, and there were some cases with blood flow increase during stimulation.

The duration of increased blood flow after the stimulation of the hippocampus was longer than that of MRF. Then, the relation between changes in EEG and blood flow
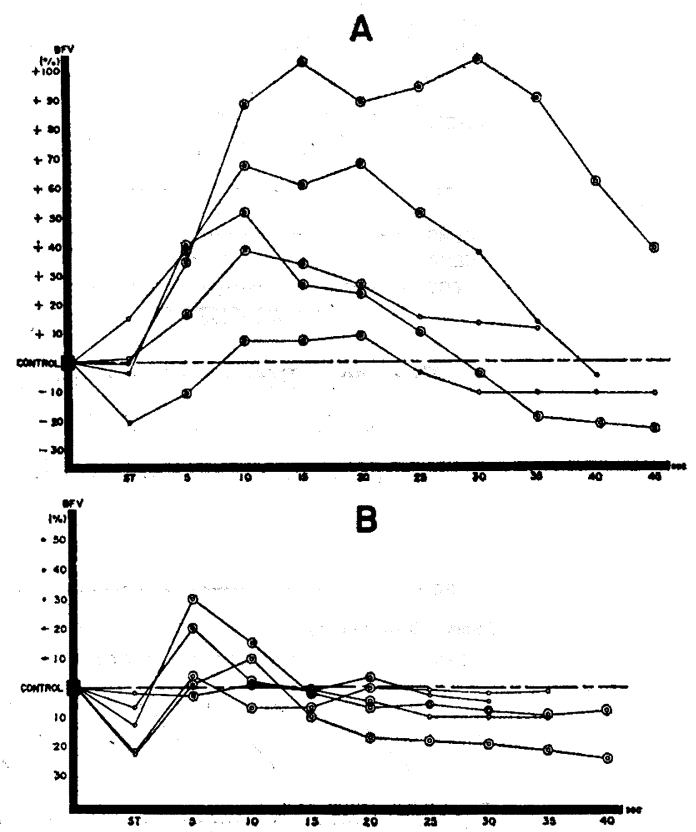

Fig. 29. Effect of stimulation of the hippocampus on carotid blood flow.

$\odot$ : When after-discharge due to stimulation of the hippocampus involved the cerebral cortex. (0): When after-discharge was localized in the hippocampus. 
$\mathrm{S}-38$

due to HPC stimulation was examined. One example is shown in Fig. 28. First, carotid blood flow decreased, then after the stimulation, carotid blood flow increased and after-discharge was observed in the cerebral cortex and the hippocampus. Then, blood flow had continued to increase until the after-discharge stopped. Blood flow showed no change while after-discharge was observed in the hippocampus. These cases were collected in Fig. 29. In Fig. 29, $\mathbf{A}$, the cases, in which after-discharge involved the cerebral cortex, were collected, and in Fig. 29, B, the cases, in which afterdischarge was localized in the hippocampus, were collected. From these results, it was

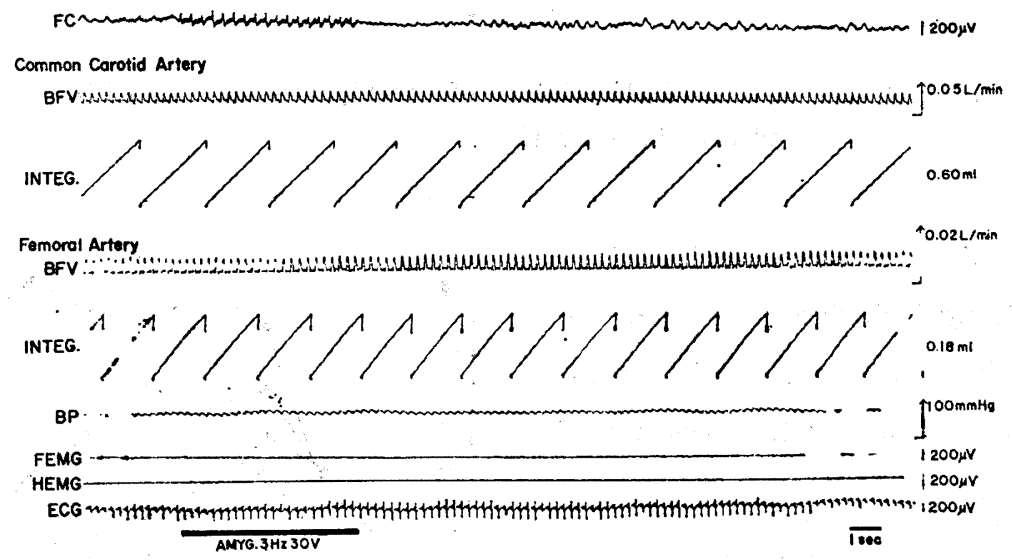

Fig. 30. Effect of stimulation of the amygdala at $3 \mathrm{~Hz}$ on EEG, carotid and femoral blood flow, arterial blood pressure, EMG and ECG.

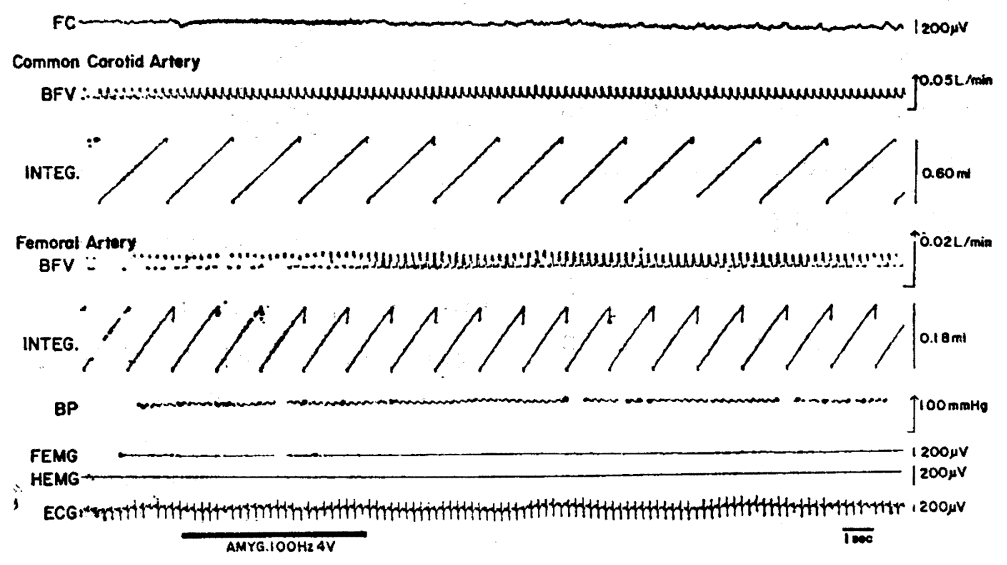

Fig. 31. Effect of stimulation of the amygdala at $100 \mathrm{~Hz}$ on EEG, carotid and femoral blood flow, arterial blood pressure, EMG and ECG. 

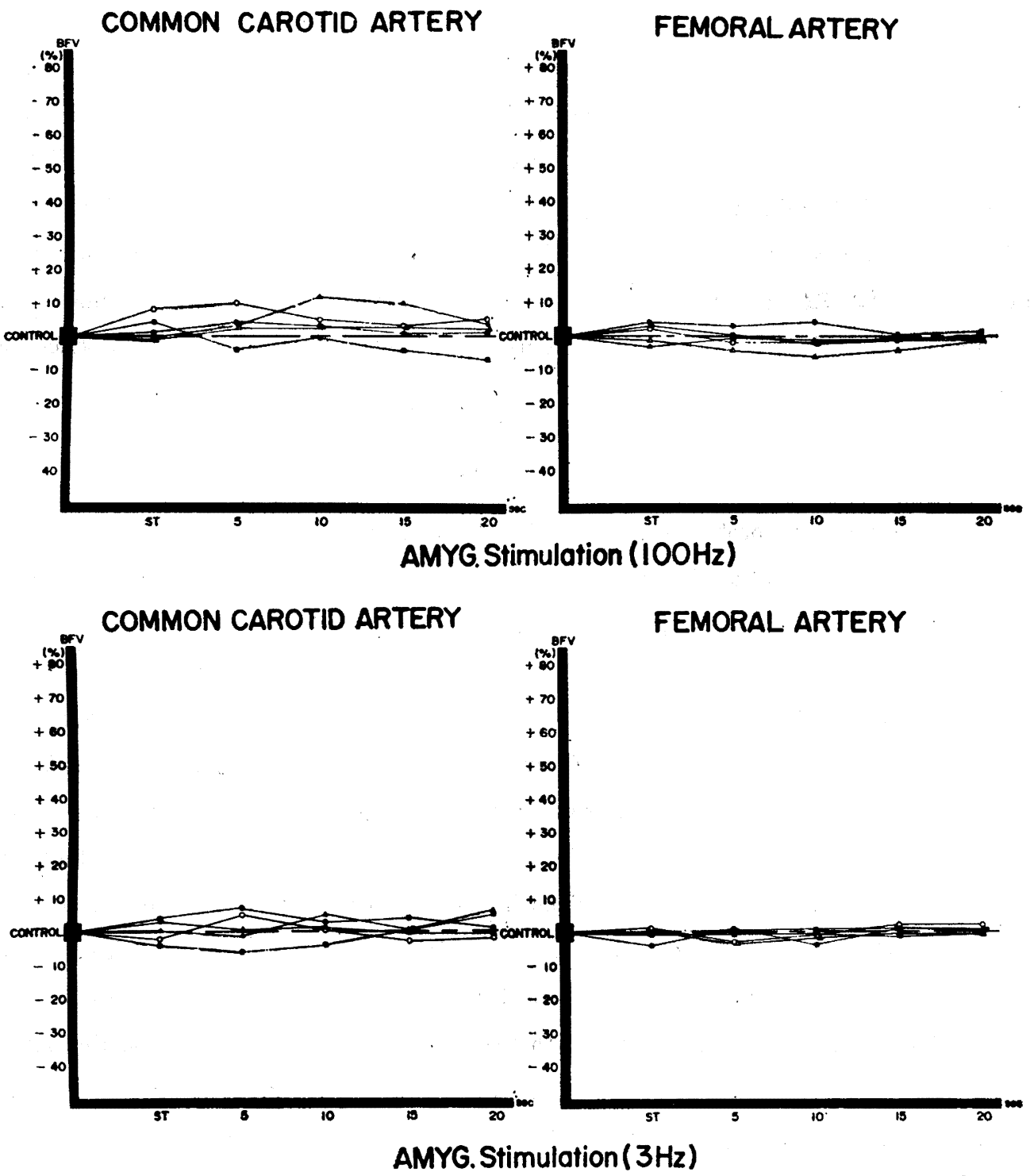

Fig. 32. Effect of stimulation of the amygdala on carotid and femoral blood flow.

understood that carotid blood flow generally increased when after-discharge due to HPC stimulation involved the cerebral cortex, and that carotid blood flow regressed to the control level when after-discharge was localized in the hippocampus.

T. Effect of stimulation of the amygdala on carotid blood flow

Effect of stimulation of the amygdala, which belongs to the limbic system, was examined. Carotid blood flow decreased temporarily due to the stimulation which produced the muscular discharge and then blood flow increased after stimulation similar to the results of MRF stimulation. Carotid blood flow did not increase when after- 
discharge due to stimulation of the amygdala was observed contrary to the results of HPC stimulation. Fig. 30 shows a case of stimulation of the amygdala at $3 \mathrm{~Hz}$ in which muscular discharge had not been observed and neither carotid blood flow, femoral blood flow, arterial blood pressure nor ECG showed any changes. Fig. 31 shows a case of stimulation of the amygdala at $100 \mathrm{~Hz}$ below the threshold of muscular discharge. There were no changes in the cardiovascular system. These cases were collected in Fig. 32. From these results it was understood that the cardiovascular system showed no change due to stimulation of the amygdala which did not produce any muscular discharge.

\section{Discussion}

A. Where to place the probe to measure the blood flow

In the present study, the focus was made upon the prompt changes in cephalic and cerebral blood flow. Therefore, electromagnetic blood flowmeter and crossed thermocouple tissue blood flowmeter were used to obtain them. Concerning the area where the probe is placed, internal carotid artery and vertebral artery are considered to be adequate when electromagnetic blood flowmeter is used, but it may not be possible to place the probe directly on the internal carotid artery of such animals as rabbits, keeping the cervical sympathetic nerve intact. It is clear that even in the monkey, the surgery necessary to implant flow devices in the internal carotid arteries can lead to interruption of reflex pathways and loss of reactivity of cerebral vessels (Dumke and Schmidt $\left.{ }^{12}\right)$. Therefore, the probe was placed on the common carotid artery to measure its blood flow. Then it was compared with the carotid blood flow after the ligation of external carotid artery and internal carotid artery respectively, and similar responses were observed. Accordingly, many experiments were performed measuring the common carotid blood flow for the sake of convenience, and the results were compared whenever necessary with that of vertebral blood flow, $r-\mathrm{CoBF}$ and carotid blood flow after the ligation of external carotid artery. In the study of pursuing the relationship between CBF and EEG, electromagnetic blood flowmeter is an effective device for measuring the prompt changes in blood flow which appears shortly after the changes in EEG. On the contrary, it may not be possible to measure the cerebral blood flow directly by use of magnetic flowmeter. Therefore, we have no other reason but to be pleased with the above mentioned methods to estimate the cerebral blood flow.

B. Carotid blood flow and femoral blood flow

A number of attempts ${ }^{13}$-15) have been made to record the carotid and femoral blood 
flow simultaneously, but there have been no reports dealing with the relations among hippocampal arousal reaction, carotid blood flow and femoral blood flow. In the present study, carotid blood flow decreased, femoral blood flow increased, arterial blood pressure increased and heart rate decreased during the presence of hippocampal theta rhythm activity. This increase in femoral blood flow can be considered to be passively produced from the result of reduction of carotid blood flow, but it seems to be the result of direct effect from the cardiovascular center, considering the unsettled relations* between carotid blood flow and femoral blood flow during the presence of desynchronization and mixed pattern in the hippocampus (*one case with increase in both carotid and femoral blood flow, one case with decrease in both carotid and femoral blood flow, one case with increase in femoral blood flow and decrease in carotid blood flow, one case with no change in femoral blood flow and increase in carotid blood flow). Since the hippocampal theta rhythm activity is considered to be related with sympathetic activity, the increase in femoral blood flow might be the result of the activity of the sympathetic cholinergic vasodialator ${ }^{16}$ ) in order to increase the skeletal muscular blood flow which allows rabbits to evade danger.

C. Cephalic blood flow during the presence of arousal reaction

It is generally assumed that the cerebral blood flow increases when arousal reaction is observed, based on numerous studies ${ }^{10) 17)-21}$ dealing with the relation between EEG and CBF. In the present study, blood flow also increased during the presence of arousal reaction, but when observed accurately, many cases with temporal blood flow reduction were noticed. As previously described, this temporal reduction was observed in the experiments in which the common carotid arteries were bluntly dissected by only $8 \mathrm{~mm}$ in length when probe was placed. Accordingly, the cause of this reduction was considered in the following manner.

Firstly, whether there is an error of measurement or not, namely the angle of probe to the artery became unstable due to the motion of rabbit during the presence of arousal reaction. This question was ruled out by the attentive and recurrent observation in which the reduction was confirmed by weak stimulation (this weak RF stimulation did not induce the muscular discharge), and also the same reduction was confirmed in the inmobilized rabbits by gallamine. Therefore this convinced me that it was not the result from the erroneous measurement. Secondly, in what area does this reduction occur ......in the internal carotid area?, external carotid area? or in both of them? In order to elucidate, carotid blood flow after the ligation of external carotid and internal carotid arteries were measured respectively, and the reduction was observed in both of 
them. Consequently, the reduction is considered to occur in the whole common carotid area. Thirdly, the vertebral blood flow was measured because the role of internal carotid artery toward brain is relatively little in rabbit, and the reduction was also observed in this blood flow during the presence of arousal reaction. Then, the reduction was also observed in the regional cortical blood flow which was measured by use of crossed thermocouple tissue blood flowmeter. Thus, in the present study, the reduction was observed in two arteries which irrigate the brain and in the cortical blood flow. However, this reduction was rarely reported in other papers. Suzuki et $\mathrm{al}^{22)}$ reported the small and prolonged reduction in cortical blood flow of cats in several cases which was measured by use of thermistor, but in most papers, the reduction of blood flow has not been reported.

Then an investigation continued further, and three conditions were found to be necessary to obtain the temporal reduction. First condition is to use the instrument which is able to obtain the prompt changes in the blood flow. It would not be recorded, if such methods as drop count, measurement of venous outflow using electromagnetic blood flowmeter, hydrogen clearance, nitrous oxide clearance, heat clearance, ${ }^{85} \mathrm{Kr}$ clearance and ${ }^{133} \mathrm{Xe}$ clearance were used, because the temporal reduction which lasts a few seconds is balanced by the rapid increase in blood flow which occurs shortly after reduction. Second condition is to refrain from using either muscle relaxant or anesthetics, namely to perform the experiments in arousal state, because in the present study, the temporal reduction disappeared following the administration of small amount of barbiturate. This could be the reason why the reduction was not observed either in the studies of Meyer or Langfitt. Third condition is to keep the cervical sympathetic nerve intact. This is clearly proved by the different results of the former half and the latter half of my study. This difference came from the difference of length of blunt dissection of the common carotid artery. The results of experiments of cervical sympathetic nerve cut also underline the importance of this nerve on the carotid blood flow. Thus, three conditions are considered to be necessary to obtain the temporal reduction of blood flow. That is why there have been few studies available which report the reduction of blood flow.

A recent study by Manrique et $\mathrm{al}^{23}$ ) reported reduction of blood flow during MRF stimulation measured by electromagnetic blood flowmeter at the internal maxillary artery of goat which gives the unique arterial blood supply to the brain. However, in their study, neither cortical nor hippocampal EEG, which are related with the changes in the blood flow, were recorded and there was no blood flow increase after the end 
of MRF stimulation, which differs from my case. This difference might come from the difference of animal species and difference of area where the probe was placed.

D. Relations between hippocampal EEG and cephalic blood flow

Torii ${ }^{8)}$ indicated that hippocampal theta rhythm activity is related with sympathetic activity and that desynchronization with parasympathetic activity based on the relations between hippocampal EEG and arterial blood pressure. The results of my study are supportive of his hypothesis, admitting a little difference between the two. During the presence of theta rhythm activity, blood pressure increased in both Torii's and mine, but during the presence of desynchronization, it decreased in Torii's study while it showed no change in mine. The cause of this difference might come from whether the anesthetics is used or not, as pointed out by Koikegami ${ }^{24)}$.

Then, it was scrutinized whether his hypothesis is proved or not by the present study. In the present study, during the presence of theta rhythm activity, carotid and vertebral blood flow decreased temporarily, r-CoBF slightly decreased in most cases and blood pressure increased. Now, generally speaking, blood flow of such arteries as carotid and vertebral arteries, which supply blood to the brain, may not be readily reduced by the activity of the sympathetic nerve controlling those arteries, because it is not physiological that the sympathetic activity invites the reduction of blood flow which may cause syncope in animal confronting danger. However, it may be natural to consider that the hyperactivity (hyperfunction) of the sympathetic nervous system resulted in the reduction of $\mathrm{CBF}$, due to the direct stimulation of the reticular formation. This mechanism of $\mathrm{CBF}$ reduction may be one factor of the syncope in man and animal having extreme excitement or astonishment. Then, taking into account the results of the experiments of cervical sympathetic nerve cut, which resulted in a considerable increase in blood flow from the temporary reduction of the control, this temporary reduction can be considered to be related with the sympathetic activity. Therefore, hippocampal theta rhythm activity which is related with the reduction of blood flow can be considered to be related with the sympathetic activity as indicated by Torii ${ }^{8)}$ and Yamamoto et $\mathrm{al}^{\text {9). }}$.

On the other hand, the question whether hippocampal desynchronization is related with the parasympathetic activity or not was not elucidated, for experiments to clarify the mechanism of the blood flow increase during the presence of desynchronization were not performed, while those of IPN lesion and administration of atropine were performed in order to clarify the mechanism of increase in carotid blood flow due to MRF stimulation during the cervical sympathetic nerve had been cut off. 
Now, a few comments on the hippocampal desynchronization will be conducted. First of all, histological difference between the sites of stimulation in the reticular formation which induced desynchronization and theta rhythm activity was not noticed, which turned out to be the same result as that of Fujimoto ${ }^{25)}$. As to the hippocampal EEG, Anchel and Lindsley ${ }^{5}$ indicated that there are two systems: one mediating the theta rhythm and the other the desynchronization pattern which involve pathways that are clearly separated in the hypothalamus. As these pathways course caudally, they appear to come closer together, and the pathways seem to converge upon closely adjacent area in the dorso-lateral region of the mesencephalic reticular formation. They also indicated that stimulation of lateral hypothalamic pathway cause hippocampal desynchronization, and in contrast, stimulation of medial hypothalamic pathway produced hippocampal theta rhythm activity. In the present study, stimulation of the medial hypothalamus readily produced hippocampal theta rhythm activity, but stimulation of the lateral hypothalamus did not readily produce hippocampal desynchronization. Paiva et $\mathrm{al}^{\left({ }^{6}\right)}$ reported that desynchronization (EEG flat spectrum) did not take place at low stimulation strength of the MRF, LH and MH except in a few cases, where the electrodes were placed in the neighbourhood of fiber tracts (fornix and medial forebrain bundle), and evidence for the existence of "synchronizing" and "desynchronizing" system was not found, and they also indicated that desynchronization was a result of too strong stimulation. However, in the present study, a case with desynchronization due to stimulation at 1 to $2 \mathrm{~V}$, changing into theta rhythm activity with the increase of strength of stimulation at $4 \mathrm{~V}$ or more, did not allow me to consider the desynchronization as the result of highly strong stimulation. Then, these unsettled results of various researchers were considered as follows. The studies of Anchel et al and Torii reporting desynchronization due to LH stimulation were performed following the administration of anesthetics while those of Paiva et al and the present study were performed without using them. Paiva et al started the chronic phase of their experiments 10 days after the operation of indwelling electrodes. This difference led me to think anesthetics as one factor of these unsettled results.

Now, a short summarization will be conducted before conclusion, as to desynchronization. 1. Hippocampal desynchronization is induced by MRF stimulation showing no special localization in the site of stimulation. 2. Desynchronization due to LH stimulation may become readily induced following the administration of anesthetics. 3 . Desynchronization may be affected by the strength of stimulation to some extent. Then, the cases with theta rhythm activity changing into desynchronization with the lapse of 
time were noticed. Consequently, experiments of ICP increase were performed considering the physical condition of animal as one factor which alters the pattern of hippocampal EEG. With the increase of ICP, hippocampal EEG changed from theta rhythm activity to mixed pattern, then to desynchronization, and with the change of hippocampal EEG, response of carotid blood flow to MRF stimulation changed, that is to say, blood flow decreased temporarily during the presence of theta rhythm activity, and changed into increase with the change of pattern of hippocampal EEG. Furthermore, there will be added one item to the above summarization. 4. Hippocampal desynchronization is readily produced with the increase of ICP.

Now, if the tonus of cerebral arteries are presumed to be regulated by the sympathetic nerve, mechanism of the alteration of hippocampal EEG from theta rhythm activity to desynchronization with the increase of ICP, may be explained as follows. With the increase of ICP, systemic arterial pressure increases (Cushing reflex) in order to prevent the decrease in $\mathrm{CBF}$. At the same time, sympathetic nerve which maintains tonus of cerebral arteries decreases its activity. Accordingly, hippocampal theta rhythm activity related with sympathetic activity diminishes, and hippocampal desynchronization emerges with increase in carotid blood flow. However, it is yet to be known that desynchronization and blood flow increase emerge simply as a result of the pause of sympathtic activity, or emerge as a result of the other nervous activity.

This chapter, as to hippocampal EEG and blood flow, will be summarized as follows. Carotid, vertebral and regional cortical blood flow decreased temporarily during the presence of hippocampal theta rhythm activity. Those are considered to be related with sympathetic activity. However, no elucidation has been made as to mechanism as well as physiological significance of hippocampal desynchronization accompanied by blood flow increase, leaving the question unsettled as posed by Torii whether or not the parasympathetic activity would be responsible for it. Hence, studies dealing with the relations between autonomic nervous system and hippocampal desynchronization is, hereafter, desired together with studies in terms of the relations between behaviour and hippocampal $\mathrm{EEG}^{\left.2()^{-34}\right)}$, role of septum on hippocampal $\mathrm{EEG}^{35)}$, hypothalamus

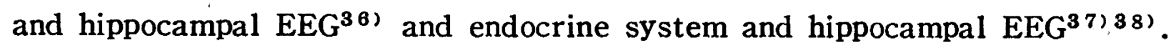

E. Autonomic influence on carotid and cortical blood flow

There is an unanimous agreement that metabolic effects are quite important and that autonomic effects are quantitatively small on CBF. However, many reports in terms of both physiology $y^{39)-42}$ and morphology $42-45$ ) have been made dealing with the neurogenic control of CBF. The reports previous to 1970 were well summarized by 
Nelson and Rennels $\left.{ }^{46}\right)$. Therefore, the reports following that year will be summarized herein.

Firstly, morphological studies are as follows. Using the fluorescence histochemical technique and electron microscopy, Iwayama ${ }^{47}$ demonstrated a dual adrenergic and nonadrenergic innervation of the anterior cerebral arteries in rats, and that this nonadrenergic fiber, which contained exclusively small agranular vesicles, could be regarded as cholinergic based on the studies by Moffat ${ }^{48)}$ and Graham ${ }^{49}$ ). Nielsen et $\mathrm{al}^{50)}$ demonstrated adrenergic and cholinergic innervation of cerebral arteries in cats by electron microscopy and histochemical technique. Licata et al ${ }^{51}$ ) also demonstrated adrenergic and cholinergic innervation of cerebral artery.

A number of physio-pharmacological studies were suggestive of the existence of adrenergic and cholinergic innervation. Evidences for sympathetic innervation of the cerebral arteries are as follows. Kobayashi et $\mathrm{al}^{52)}$ reported constriction of the superficial arterial vessels and decrease in CoBF due to stimulation of the cervical sympathetic nerve in cats. Traystman et $\mathrm{al}^{53}$ ) reported decrease in cerebral venous outflow and carotid flow due to stimulation of the stellate ganglion in dogs. Besides them, Harper et $\mathrm{al}^{54)}$ and $\mathrm{D}^{\prime}$ Alecy et $\mathrm{al}^{55)}$ indicated evidences of sympathetic regulation of $\mathrm{CBF}$. Then, the evidences for the cholinergic innervation are as follows. Kawamura et $\mathrm{al}^{56}$ ) indicated that a central vasodilator tonus of the cerebral vessels was considered to be maintained by innervation of the cerebral vessels : cholinergic neurons which have their central origin in the brain stem and diencephalic area based on the results of intravertebral injection of atropine which suppressed the increase in cerebral venous outflow by inhalation of $5 \% \mathrm{CO}_{2}$, and also based on the fact that the vertebrobasilar arterial system supplies the brain stem and diencephalon. Scremin et $\mathrm{al}^{57}$ ) indicated that the increase in cortical blood flow, measured by hydrogen clearance method, which accompanies cortical desynchronization in the urethanized rats, was mediated, at least in part, by a neurogenic mechanism that involves a cholinergic step at the cortical level, based on the results of the topical application of atropine which prevented the increase in blood flow during the presence of cortical desynchronization in stage 3 of urethan anesthesia, and based on the results of application of arecoline, carbaminoylcholine, pilocarpine or acetylcholine with eserine which increased cortical blood flow significantly. Aoyagi et $\mathrm{al}^{58}$ ) indicated that central cholinergic cerebrovascular influences may exist and are vasodilatory in nature, based on the results of intravertebral administration of neostigmine which significantly enhanced the cerebral vasodilatory reactivity to $\mathrm{CO}_{2}$ inhalation. Furthermore, Kuschinsky et $\mathrm{al}^{59)}$ indicated the existence of cholinergic 
receptors in pial arteries, based on the results of application of carbachol into the perivascular space which led to vasodilation. Thus, as above summarized, a number of studies have suggested the existence of adrenergic and cholinergic regulation of cerebral arteries.

Now, in the present study, the changes in cephalic blood flow due to MRF stimulation were affected by the cervical sympathetic nerve, and so were the changes in $\mathrm{r}-\mathrm{CoBF}$. Namely, decrease or no change in cephalic and cortical blood flow turned into considerable increase by the cervical sympathetic nerve cut. Therefore, the temporary reduction of cephalic and cortical blood flow due to MRF stimulation is considered to be mediated by the cervical sympathetic nerve.

Then, the mechanism of the rapid increase in blood flow due to MRF stimulation, during the cervical sympathetic nerve had been cut off, was examined whether it is metabolic or neurogenic (except the cervical sympathetic nerve) in the following manner. If it is metabolic, the latency of one to two seconds seemed to be too fast. However, blood gases had already been changed during stimulation: $\mathrm{PaO}_{2}$ increased, $\mathrm{PaCO}_{2}$ decreased and $\mathrm{pH}$ increased. Therefore, the metabolic influence could not be ruled out. Accordingly, this problem needs further elucidation. Then, in order to examine the neurogenic influences, the experiments of IPN lesion, IPN stimulation and atropine administration were performed. Kuhar et $\mathrm{al}^{60}$ ) indicated that the most established and studied cholinergic tract in the brain is the septal-hippocampal pathway, and that some of the habenulo-interpeduncular neurons are probably cholinergic. They also indicated that the latter neurons might have pharmacological and functional differences compared with the former neurons. Then, at first, the experiments were performed in which impairment of septal-hippocampal pathway was involved, but no significant changes were noticed in the increase in blood flow due to MRF stimulation. Following this, the experiments of impairment of IPN, which belongs to the latter neurons of habenulointerpeduncular neurons and contains a great amount of choline acetyltransferase (an important enzyme for the synthesis of acetylcholine) as referred to by Palkovits ${ }^{61}$, were performed. The increase in carotid blood flow due to MRF stimulation with the cervical sympathetic nerve cut of was inhibited by the impairment of IPN. The same result was confirmed in $\mathrm{r}-\mathrm{CoBF}$. Therefore, it might be indicated that cephalic blood flow and $r-\mathrm{CoBF}$ are regulated by cholinergic neurons, based on my results together with the Kawamura's hypothesis that the cholinergic neurons, which maintain the vasodilator tonus, have their central origin in the brain stem and diencephalic area. The results of experiments of atropine also suggest the existence of cholinergic control. They alone 
are, however, inadequate to reach the conclusion, because effects of atropine are not limited to the neurons which control the cephalic and cerebral arteries, even though no changes were noticed either in arterial pressure or on ECG following the administration of them. Increase in blood flow due to IPN stimulation was unexpectedly small compared with the results of impairment of IPN. It might be considered that the neurons, which were not impaired in the experiments of IPN lesion, were stimulated. Therefore, as to the effect of IPN on cephalic and cerebral blood flow, further elucidation is desired.

Now, based on the present study, it is indicated that there might exist both sympathetic and cholinergic (which is related with IPN) influences on cephalic and cortical blood flow.

F. Cephalic blood flow during the presence of recruiting response and spike and wave Morison and Dempsey ${ }^{62}$ ) indicated that recruiting response was produced by stimulation of the diffuse thalamo cortical projection system. Following this report, many researchers (Weinberger et $\mathrm{al}^{63}$ ), Watanabe ${ }^{64)}$, Hayashi ${ }^{65)}, \mathrm{Handa}^{68)}, \mathrm{Nasu}^{67)}$ ) attempted to reveal the functional significances of this response. Yasuhara ${ }^{68}$ ) indicated that the afferent pathways were inhibited during the presence of spike and wave, that spikes of spike and wave were transformed into recruiting response with the increase of the frequency of stimulation in the thalamus (VA, CM), that the afferent pathways were also inhibited by recruiting response, and that the arousal reactions were inhibited by recruiting response. Based on those results, he indicated that both spike and wave and recruiting response were related with the inhibitory mechanism in the central nervous system.

As to the effects of recruiting response and spike and wave on the autonomic system, Ichiki ${ }^{(9)}$ and Mori ${ }^{70}$ reported the effects on intestinal movement, and Uchiyama ${ }^{10)}$ reported the effects of recruiting response on carotid blood flow in which some cases of the blood flow decrease were noticed during the presence of that response when muscular discharges did not prevail. Suzuki et al ${ }^{71}$ ) reported the decrease in $r-C o B F$ during the presence of recruiting response in cats. In the present study, there were also some cases of carotid blood flow decrease during the presence of recruiting response.

This decrease in carotid and cortical blood flow may be explained by the results of Yasuhara's studies. That is to say, the blood flow may decrease reflecting the attenuation in the brain activity due to the inhibitory system activity which is related with the recruiting response, contrasting the blood flow response to the arousal reaction which 
is considered to increase. These decreases were, however, extremely slight even if they occurred and no changes in blood flow were noticed during the presence of spike and wave. These results lead me to consider that stimulation of the thalamus at a low frequency scarcely affected the carotid blood flow, and that the inhibitions of the central nervous system during the presence of recruiting response or spike and wave were not produced passively by the reduction in the carotid blood flow. Finally, there may exist the functional differences between spike and wave and recruiting response based on the differences in the blood flow responses to them.

G. Effects of pentobarbital and metrazol on carotid and femoral blood flow

Pentobarbital reduces the oxygen consumption in the brain, and this reduction is caused by the inhibition of the brain activity (Homburger ${ }^{72)}$, Himwich ${ }^{73}$ and Wechsler ${ }^{74}$ ). Goldman $^{75)}$ indicated that pentobarbital reduced blood flow of the hypothalamus, basal ganglia, midbrain, dorsal hippocampus and the cortex. Besides this report, many researchers ${ }^{73)}$ 76)-79) reported decrease in CBF. On the contrary, a paper ${ }^{74)}$ on blood flow increase and papers ${ }^{80)}$ 81) $^{\text {) }}$ on no change in blood flow have also been made. In the present study, numbers of increased and decreased blood flow cases were even. Taking into account the results of the femoral blood flow increase following the administration of pentobarbital, there may be a discrepancy in the carotid blood flow : external carotid blood flow increased while internal carotid blood flow decreased. Then, the changes in carotid and femoral blood flow due to MRF stimulation were inhibited by the administration of $5 \mathrm{mg} / \mathrm{kg}$ pentobarbital. This inhibition on the blood flow could be considered as the results of inhibitory effect of pentobarbital on the reticular formation, based on the Fujimoto's study ${ }^{25}$ ) which indicated the increase in threshold of the arousal reaction due to MRF stimulation following the administration of pentobarbital.

Metrazol has been widely studied in man and experimental animals. All levels of the cerebrospinal axis are stimulated by the drug. In the present study, with the administration of metrazol, muscular discharge spontaneously emerged. Then, blood flow fluctuated in the presence of muscular discharge. Following the administration of 5 $\mathrm{mg} / \mathrm{kg}$ or $3 \mathrm{mg} / \mathrm{kg}$ metrazol, increases in carotid blood flow due to MRF stimulation were slightly facilitated, but following the administration of $20 \mathrm{mg} / \mathrm{kg}$ metrazol, blood flow stopped changing. This reduction of reactivity may not have occurred either due to the inhibition of the reticular formation by metrazol or due to the dilatation of the arteries, for in the Fujimoto's study which investigated effects of metrazol on the arousal reaction, he indicated the threshold of the arousal reaction decreased following 
the administration of $20 \mathrm{mg} / \mathrm{kg}$ metrazol. Furthermore, in the present study, the absolute values of carotid and femoral blood flow did not always increase following the administration of $20 \mathrm{mg} / \mathrm{kg}$ metrazol. Consequently, the cause of this reduced responses of carotid and femoral blood flow to MRF stimulation was considered as follows. Somewhere in the tract from the reticular formation to the carotid or femoral artery, inhibitory systems toward vasoconstrictor were inhibited, vasodilators were inhibited or inhibitory systems toward vasodilator were activated. Lewin and Esplin ${ }^{82)}$ have suggested that metrazol stimulates excitatory and inhibitory neurons. Therefore, it is considered that there may exist the inhibitory system toward vasodilator and that this system may be activated by metrazol resulting in the reduced response to MRF stimulation.

H. Cephalic blood flow and limbic system

The stimulation of the limbic system usually produces the autonomic responses. Stimulation of the hippocampus (HPC) at high frequency produces after-discharges in the limbic system and cerebral cortex. $A n d y^{83)}$ reported that blood pressure depression developed during the course of HPC stimulation or at the onset of a discharge and that blood pressure elevations usually developed in the late phase of a discharge and most frequently occurred with the longer after-discharges. Nishino ${ }^{84)}$ reported the after-discharge which continued 40 seconds in the cerebral cortex and amygdala due to HPC stimulation and that 10 to 15 seconds after the beginning of the first change in EEG, blood flow of the contralateral hippocampus, measured by use of double thermistor, began to increase lasting 2 minutes. In the present study, relation among after-discharge due to HPC stimulation, arterial pressure and cephalic blood flow was investigated. Blood flow increase was observed corresponding with the presence of after-discharge which involved the cerebral cortex, in contrast with the blood flow which showed no change when after-discharge was localized in the hippocampus. In the study of Nishino, after-discharge in the hippocampus was not recorded, therefore it is not known whether or not the blood flow increase that continued 2 minutes in the hippocampus was accompanied by the after-discharge which involved the hippocampus. However, it was not until 10 to 15 seconds after the first change had taken place in EEG that the hippocampal blood flow started to increase, and it continued to increase for a while after the regression of changes in EEG. Accordingly, it is considered that the sensitivity of the device for measurement of blood flow (double thermistor) might not be sharp enough.

Now, it is indicated that the hippocampal blood flow may increase during the 
presence of after-discharge localized in the hippocampus, but cephalic blood flow is not affected by such after-discharge that does not involve the cerebral cortex.

Autonomic responses are readily produced by stimulation of the amygdala (Koikegami 24) ), though, in the present study, no changes in either cephalic blood flow or arterial pressure in spite of stimulation of the amygdala were noticed. There are, however, only 5 cases. Accordingly, as to the effects of the amygdala on the autonomic responses, further elucidation is desired.

\section{Summary}

1. During stimulation of the mesencephalic reticular formation (MRF), temporary decreases in carotid, vertebral and cortical blood flow were observed on rather many occasions. Such decreases were found to be closely related with the hippocampal theta rhythm activity, while increases in blood flow during MRF stimulation were closely related with the hippocampal desynchronization.

2. When intracranial pressure increased, EEG pattern of the hippocampus during MRF stimulation changed from theta rhythm activity to desynchronization, accompanied by a change in the carotid blood flow from decrease to increase.

3. Decrease in carotid blood flow during MRF stimulation changed to increase due to an incision of the cervical sympathetic nerve (CSN), and this increase in blood flow was reduced following the administration of atropine. Furthermore, the interpeduncular nucleus had a relation with the changes in carotid blood flow due to MRF stimulation.

4. Carotid blood flow in the presence of recruiting response and spike and wave remained almost unchanged.

5. When after-discharge due to stimulation of the hippocampus involved the cerebral cortex, carotid blood flow generally increased.

6. Stimulation of the amygdala, which did not induce the muscular discharge, produced no changes in carotid blood flow.

7. There were cases with both increase and decrease in femoral blood flow during MRF stimulation. The sites of stimulation which caused decrease concentrated on the center of the reticular formation of P. 8 according to Sawyer's atlas.

8. Pentobarbital inhibited the changes in carotid and femoral blood flow due to MRF stimulation, and it increased the femoral blood flow.

9. Number of the cases of the muscular discharge increased following the administration of metrazol, at which time, carotid and femoral blood flow increased. 
An outline of the present results was reported at the 9th International Congress of EEG and Clinical Neurophysiology, at the 7th Japanese Congress of EEG and EMG, at the 54th Japanese Congress of Physiology and at the 53rd Japanese Congress of Physiology.

The auther wishes to express his thanks to Prof. M. Yasuhara for valuable advice and strong encouragement during the course of the present study. Thanks are also tendered to Associate Prof. H. Naito for her earnest guidance and to colleagues for many helpful discussions.

\section{References}

1) Roy, C.S. and Sherrington, C.S. : On the regulation of the bloo-dsupply of the brain. J. Physiol., 11, 85, 1890.

2) Green, J. D. and Arduini, A. A. : Hippocampal electrical activity in arousal. J. Neurophysiol., 17, 533, 1954.

3) Stumpf, C. : The fast component in the electrical activity of rabbit's hippocampus. EEG Clin. Neurophysiol., 18, 477, 1965.

4) Yokota, T. and Fujimori, B. : Effects of brain-stem stimulation upon hippocampal electrical activity, somatomotor reflexes and autonomic functions. EEG Clin. Neurophysiol., 16, 375, 1964.

5) Anchel, H. and Lindsley, D.B. : Differentiation of two reticulo-hypothalamic systems regulating hippocampal activity. EEG Clin. Neurophysiol., 32, 209, 1972.

6) Paiva, T., Lopes da Silva, F.H. and Mollevanger, W. : Modulating systems of hippocampal EEG. EEG Clin. Neurophysiol., 40, 470, 1976.

7) Klemm, W.R. : Hippocampal EEG and information processing : A special role for theta rhythm. Prog. Neurobiol., 7, 197, 1976.

8) Torii, s.: Two types of pattern of hippocampal electrical activity induced by stimulation of hypothalamus and surrounding parts of rabbit's brain. Jap. J. Physiol., 11, 147, 1961.

9) Yamamoto, K. and Kido, R. : Hippocampal arousal wave as an indicator of electrical activities of the brain. Brain and Nerve, 13, 887, 1961.

10) Uchiyama, S. : An electrophysiological study on relations between the activity of the central nervous system and cerebral circulation. J. Kansai Med. Univ., 28, 380, 1976.

11) Sawyer, C.H., Everett, J.W. and Green, J.D. : The rabbit diencephalon in stereotaxic cordinates. J. Comp. Neurol., 101, 801, 1954.

12) Dumke, P.R. and Schmidt, C.F. : Quantitative measurements of cerebral blood flow in the macacque monkey. Am. J. Physiol., 138, 421, 1943.

13) Hallberg, D. and Pernow, B. : Effect of substance $P$ on various vascular beds in the dog. Acta. Physiol. Scand., 93, 277, 1975.

14) Reddy, G. D., Gootman, N., Buckley, N. M., Gootman, P. M. and Crane, L. : Regional blood flow changes in neonatal pigs in response to hypercapnia, hemorrhage and sciatic nerve stimulation, Biol. Neonate., 25, 249, 1974.

15) Hallberg, D., Andreen, M, Odensjö, G. and Soda, M. : Cardiac output and regional blood 
flows during intravenous infusion of an amino acid mixture in dogs. IV. Acta. Chir. Scand., 140, 441, 1974.

16) Folkow, B., Uvnäs, In B. : Sympathetic vasodilator system and blood flow; discussion. Physiol. Rev., 40, Suppl. 4, 77, 1960.

17) Darrow, C. W. and Graf, C. G. : Relation of electroencephalogram to photometrically observed vasomotor changes in the brain. J. Neurophysiol., 8, 449, 1945.

18) Ingvar, D.H. and Söderberg, U. : Cortical blood flow related to EEG patterns evoked by stimulation of the brain stem. Acta Physiol. Scand., 42, 130, 1958.

19) Langfitt, T.W. and Kassell, N.F. : Cerebral vasodilatation produced by brain-stem stimulation : neurogenic control vs. autoregulation. Am. J. physiol., 215, 90, 1968.

20) Meyer, J.S. et al. : Effect of stimulation of the brain-stem reticular formation on cerebral blood flow and oxygen consumption. EEG Clin. Neurophysiol., 26, 125, 1969.

21) Baldy-Moulinier, M. : Cerebral electrical activity and cerebral blood flow during brain activation. in Alfred Benzon symposium VIII "Brain work" : The coupling of function, metabolism and blood flow in the brain. edited by Ingvar, D. H. and Lassen, N. A., Munksgaard, Copenhagen, 353, 1975.

22) Suzuki, H. and Tukahara, Y. : Cerebral circulation during arousal reaction of EEG. Tohoku J. exp. Med., 84, 316, 1965.

23) Manrique, M., Alborch, E. and Delgado, J.M.R. : Cerebral blood flow and behavior during brain stimulation in the goat. Am. J. Physiol., 232, H 495, 1977.

24) Koikegami, H. : Limbic system, Chûgai lgaku Sôsho. Tokyo, 1976. (in Japanese)

25) Fujimoto, S. : An electrophysiological study on the thresholds of arousal reaction and evoked muscular discharge. J. Kansai Med. Sch., 21, 282, 1969.

26) Kemp, I.R. and Kaada, B.R. : The relation of hippocampal theta activity to arousal, attentive behaviour and somato-motor movements in unrestrained cats. Brain Res., 95, $323,1975$.

27) Gasanov, G. G. and Khanukayev, E.M. : Correlation of hippocampal theta-rhythm with behavior in rabbits. ZH. Vyssh. Nerv. Deiat., 25, 110, 1975.

28) Klemm, W.R. : Physiological and behavioral significance of hippocampal rhythmic, slow activity ("theta rhythm"). Prog. Neurobiol., 6, 23, 1976.

29) Myhrer, T. : Jump avoidance acquisition and locomotor behavior in rats with the hippoCampal theta rhythm disrupted. Behav. Biol., 19, 361, 1977.

30) Wetzel, W., Ott, T. and Matthies, H. : Hippocampal rhythmic slow activity ("theta") and behavior elicited by medial septal stimulation in rats. Behav. Biol., 19, 534, 1977.

31) Macrides, F. : Temporal relationships between hippocampal slow waves and exploratory sniffing in hamsters. Behav. Biol., 14, 295, 1975.

32) Nyakas, C. and Van Delft, A.M.L. : Behavioral and electrocortical activity in rats after neonatal intraventricular 6-hydroxydopamine administration. Pharmacol. Biochem. Behav., 3, $271,1975$.

33) Coleman, J.R. and Lindsley, D. B. : Hippocampal electrical correlates of free behavior and behavior induced by stimulation of two hypothalamic-hippocampal systems in the cat. Exp. Neurol., 49, 506, 1975.

34) Arnolds, D.E.A.T., Lopes da Silva, F.H., Kamp, A. and Aitink, J.W. : Hippocampal EEG correlates with movement in dogs. EEG Clin. Neurol., 43, 567, 1977.

35) Macadar, O., Roig, J. A., Monti, J. M. and Budelli, R. : The functional relationship 
between septal and hippocampal unit activity and hippocampal theta rhythm. Physiol. Behav., 5, 1443, 1970.

36) Wilson, C. L., Motter, B. C. and Lindsley, D. B. : Influences of hypothalamic stimulation upon septal and hippocampal electrical activity in the cat. Brain Res., 107, 55, 1976.

37) Martin, S. M., Moberg, G. P. and Horowitz, J.M. : Glucocorticoids and the hippocampal theta rhythm in loosely restrained, unanesthetized rabbits. Brain Res., 93, 535, 1975.

38) Urban, I. and Wied, D.D. : Changes in exitability of the theta activity generating substrate by ACTH 4-10 in the rat. Exp. Brain Res., 24, 325, 1976.

39) Forbes, H.S., and Wolff, H.G. : Cerebral circulation. III. The vasomotor control of cerebral vessel. Archs. Neurol. Psychiat., Chicago, 19, 1057, 1928.

40) Forbes, H. S., and Cobb, S. S. : Vasomotor control of cerebral vessels. Brain, 61, 221, 1938.

41) Forbss, H. S., Schmidt, C. F., and Nason, G.I. : Evidence of vasodilator innervation in the parietal cortex of the cat. Am. J. Physiol., 125, 216, 1939.

42) Chorobski, J., and Penfield, W. : Cerebral vasodilator nerves and their pathway from the medulla oblongata. With observations on the pial and intracerebral vascular plexus. Archs. Neurol. Psychiat., Chicago, 28, 1257, 1932.

43) McNaughton, F.L. : The innervation of the intracranial blood vessels and dural sinuses. Res. Publs. Ass. Res. nerv. ment. Dis., 18, 178, 1938.

44) Clark, S. L. : Innervation of the chorioid plexuses and the blood vessels within the central nervous system. J. Comp. Neurol., 60, 21, 1934.

45) Fang, H.C.H. et al. : Cerebral arterial innervations in man. Arch. Neurol., 4, 651, 1961.

46) Nelson, E. and Rennels, M. : Innervation of intracranial arteries. Brain, 93, 475, 1970.

47) Iwayama, T., Furness, J. B., and Burnstock, G. : Dual adrenergic and cholinergic innervation of the cerebral arteries of the rat. An ultrastructural study. Circ. Res., 26, 635, 1970.

48) Moffat, D.B. : The fine structure of the blood vessels of the renal medulla with particular reference to the control of the medullary circulation. J. Ultrastruct. Res. 19, 532, 1967.

49) Graham, J.D.P., Lever, J.D., and Spriggs, T. L. B. : An examination of adrenergic axons around pancreatic arterioles of the cat for the presence of acetylcholinesterase by high resolution autoradiographic and histochemical methods. Brit. J. Pharm. Chem. 33, 15, 1968.

50) Nielsen, K. C., Edvinsson, L. and Owman, C. : Cholinergic innervation and vasomotor response of brain vessels. in Cerebral circulation and metabolism, edited by Langfitt, T. W. et al, 473, 1975. Springer-Verlag.

51) Licata, R.H., Olson. D.R. and Mack, E.w. : Cholinergic and adrenergic innervation of cerebral vessels, in Cerebral circulation and metabolism edited by Langfitt, T.W. et al., 466, 1975, Springer-Verlag.

52) Kobayashi, S., Waltz, A.G. and Rhoton, A.L.Jr. : Effects of stimulation of cervical sympathetic nerves on cortical blood flow and vascular reactivity. Neurol., 21, 297, 1971.

53) Traystman, R. J. and Rapela, C. E. : Effect of sympathetic nerve stimulation on cerebral and cephalic blood flow, in cerebral circulation and metabolism, edited by Langfitt, T. W. et al, 451, 1975, Springer-Verlag.

54) Harper, A.M., Deshmukh, V.D., Rowan, J. O., and Jennett, W.B. : The influence of sympathetic nervous activity on cerebral blood flow. Arch. Neurol. 27, 1, 1972. 
55) D'Alecy, L. G. and Feigl, E. O. : Sympathetic control of cerebral blood flow in dogs, Circ. Res., 31, 267, 1972.

56) Kawamura, Y., Meyer, J. S., Hiromoto, H., Aoyagi, M., Tagashira, Y. and Ott, E. O. : Neurogenic control of cerebral blood flow in the baboon. J. Neurosurg., 43, 676, 1975.

57) Scremin, 0.U. et al. : Cholinergic control of blood flow in the cerebral cortex of the rat. Stroke, 4, 232, 1973.

58) Aoyagi, M., Meyer, J.S., Deshmukh, V. D., Ott, E. O., Tagashira, Y., Kawamura, Y., Matsuda, M., Achari, A.N. and Chee, A.N. C. : Central cholinergic control of cerebral blood flow in the baboon. J. Neurosurg., 43, 689, 1975.

59) Kuschinsky, W. and Wahl, M. : The functional significance of $\beta$-adrenergic and cholinergic receptors at pial arteries : A microapplication study, in Cerebral circulation and metabolism edited by Langfitt, T.W. et al, 470, 1975, Springer-Verlag.

60) Kuhar, M.J. et al. : Further evidence for cholinergic habenulo-interpeduncular neurons : pharmacologic and functional characteristics. Brain Res., 97, 265, 1975.

61) Palkovits, M., Saavedra, J.M, Kobayashi, R.M. and Brownstein, M. : Choline acetyltransferase content of limbic nuclei of the rat. Brain Res., 79, 443, 1974.

62) Morison, R.S. and Dempsey, E.W. : A study of thalamo-cortical relations. Am. J. Physiol., 135, 281, 1942.

63) Weinberger, N.M., Velasco, M. and Lindsley, D. B. : Effects of lesions upon thalamically induced electrocortical desynchronization and recruiting. EEG. Clin. Neurophysiol., 18, 369, 1965.

64) Watanabe, T. : An electrophysiological study on the inhibitory mechanism of recruiting response in the central nervous system. J. Kansai Med. Sch., 23, 467, 1971.

65) Hayashi, M. : Recruiting response and afferent pathway. J. Kansai Med. Sch., 20, 209, 1968.

66) Handa, F. : Thalamus and muscular discharge. J. Kansai Med. Sch., 19 269, 1967.

67) Nasu, C. : Electrophysiological studies of the action of cardiazol on barbiturate anesthesia, J. Kansai Med. Sch., 19, 120, 1967.

68) Yasuhara, M. : Neuropharmacological studies on arousal reaction and recruiting response. J. Kansai Med. Sch., 16, 151, 1964.

69) Ichiki, K : Electrophysiological study on the central nerve innervation of intestinal movement. J. Kansai Med. Sch., 21, 179, 1969.

70) Mori, T. : Electrophysiological analysis of thalamic induced spike and wave in rabbits. J. Kansai Med. Sch., 27, 208, 1975.

71) Suzuki, H. et al. : Regional cortical blood flow and EEG. Jap. J. EEG EMG, 5, 68, 1977. (in Japanese)

72) Homburger, E., Himwich, W.A., Etsten, B., York, G., Maresca, R. and Himwich, H.E. : Effect of pentothal anesthesia on canine cerebral cortex. Am. J. Physiol., 147, 343. 1946.

73) Himwich, W. A., Homburger, E., Maresca, R. and Himwich, H.E. : Brain metabolism in man : unanesthetized and in pentothal narcosis. Am. J. Psychiat., 103, 689, 1947.

74) Wechsler, R.L., Dripps, R.D. and Kety, S.S. : Blood flow and oxygen consumption of the human brain during anesthesia produced by thiopental. Anesthesiology, 12, 308, 1951.

75) Goldman, H. and Sapirstein, L.A. : Brain blood flow in the conscious and anesthetized rat. Am. J. Physiol., 224, 122, 1973.

76) Forsyth, R.P. and Hoffbrand, B.I. : Redistribution of cardiac output after sodium pento- 
barbital anesthesia in the monkey. Am. J. Physiol., 218, 214, 1970.

77) MeCall, M.L. and Taylor, H.W. : Effects of barbiturate sedation on the brain in toxemia of pregnancy. J. Am. Med. Assoc., 149, 51, 1952.

78) Pierce, E. C., Lambertsen, C.J., Deutsch, S. Chase, P.E. Linde, H.W., Dripps, R. D. and Price, H.L. : Cerebral circulation and metabolism during thiopental anesthesia and hyperventilation in man. J. Clin. Invest., 41, 1664, 1962.

79) Schieve, J. F. and Wilson, W. P. : The influence of age, anesthesia and cerebral arteriosclerosis on cerebral vascular activity to $\mathrm{CO}_{2}$. Am. J. Med., 15, 171, 1953.

80) Fazekas, J.F., and Bessman, A.N. : Coma mechanisms. Am. J. Med., 15, 804, 1953.

81) Wilson, W.P. et al. : The effect of carbon dioxide on cerebral blood flow, spinal fluid pressure and brain volume during pentothal sodium anesthesia. Current Res. Anesthesia Analgesia, 32, 268, 1953.

82) Lewin, J. and Esplin, D.W. : Analysis of the spinal excitatory action of pentylenetetrazol. J. Pharmacol. Exp. Ther., 132, 245, 1961.

83) Andy, 0.J. et al. : Electro-physiological comparisons of the dorsal and ventral hippocampus, in Physiologie de I'hippocampe, edited by Passouant, P., 411, 1962, CNRS, Paris.

84) Nishino, H. : Studies on the regional blood flow in cat brain. Third report. Effects of electrical stimulation on regional blood flow in certain areas of brain. Folia Pharmacol. Japon, 69, 855, 1973. 